\title{
A combined epigenome- and transcriptome-wide association study of the oral masticatory mucosa assigns CYP1B1 a central role for epithelial health in smokers
}

Gesa M. Richter ${ }^{1 *}$ (D) Jochen Kruppa ${ }^{2}$, Matthias Munz ${ }^{1,3}$, Ricarda Wiehe ${ }^{1}$, Robert Häsler ${ }^{4}$, Andre Franke ${ }^{4}$, Orlando Martins ${ }^{5}$, Yvonne Jockel-Schneider ${ }^{6}$, Corinna Bruckmannn ${ }^{7}$, Henrik Dommisch ${ }^{1}$ and Arne S. Schaefer ${ }^{1}$

\begin{abstract}
Background: The oral mucosa has an important role in maintaining barrier integrity at the gateway to the gastrointestinal and respiratory tracts. Smoking is a strong environmental risk factor for the common oral inflammatory disease periodontitis and oral cancer. Cigarette smoke affects gene methylation and expression in various tissues. This is the first epigenome-wide association study (EWAS) that aimed to identify biologically active methylation marks of the oral masticatory mucosa that are associated with smoking.

Results: Ex vivo biopsies of 18 current smokers and 21 never smokers were analysed with the Infinium Methylation EPICBeadChip and combined with whole transcriptome RNA sequencing (RNA-Seq; 16 mio reads per sample) of the same samples. We analysed the associations of $\mathrm{CpG}$ methylation values with cigarette smoking and smoke pack year (SPY) levels in an analysis of covariance (ANCOVA). Nine CpGs were significantly associated with smoking status, with three CpGs mapping to the genetic region of CYP1B1 (cytochrome P450 family 1 subfamily B member 1; best $p=5.5 \times 10^{-8}$ ) and two mapping to AHRR (aryl-hydrocarbon receptor repressor; best $p=5.9 \times 10^{-9}$ ). In the SPY analysis, $61 \mathrm{CpG}$ sites at 52 loci showed significant associations of the quantity of smoking with changes in methylation values. Here, the most significant association located to the gene CYP1B1, with $p=4.0 \times 10^{-10}$. RNA-Seq data showed significantly increased expression of CYP1B1 in smokers compared to non-smokers ( $p=$ $\left.2.2 \times 10^{-14}\right)$, together with 13 significantly upregulated transcripts. Six transcripts were significantly downregulated. No differential expression was observed for AHRR. In vitro studies with gingival fibroblasts showed that cigarette smoke extract directly upregulated the expression of CYP1B1.

Conclusion: This study validated the established role of CYP1B1 and AHRR in xenobiotic metabolism of tobacco smoke and highlights the importance of epigenetic regulation for these genes. For the first time, we give evidence of this role for the oral masticatory mucosa.
\end{abstract}

Keywords: EWAS, Methylation, Expression, Masticatory mucosa, CYP1B1, AHRR, Cytochrome P 450 pathway, OSCC, Smoking

\footnotetext{
* Correspondence: gesa.richter@charite.de

${ }^{1}$ Department of Periodontology and Synoptic Dentistry, Institute for Dental

and Craniofacial Sciences, Charité - Universitätsmedizin Berlin, Freie

Universität Berlin, Humboldt-Universität zu Berlin, and Berlin Institute of

Health, Aßmannshauser Str. 4-6, 14197 Berlin, Germany

Full list of author information is available at the end of the article
}

(c) The Author(s). 2019 Open Access This article is distributed under the terms of the Creative Commons Attribution 4.0 International License (http://creativecommons.org/licenses/by/4.0/), which permits unrestricted use, distribution, and

reproduction in any medium, provided you give appropriate credit to the original author(s) and the source, provide a link to the Creative Commons license, and indicate if changes were made. The Creative Commons Public Domain Dedication waiver (http://creativecommons.org/publicdomain/zero/1.0/) applies to the data made available in this article, unless otherwise stated. 


\section{Background}

Smoking is considered one of the most important environmental factors causing premature death by promoting a variety of malignant and non-malignant diseases. It increases the relative risk of dying by up to 2.8 and shortens lifetime expectancies by 10 years on average [1]. The oral mucosa is the barrier tissue that is most directly exposed to cigarette smoke. Accordingly, smoking is a strong risk factor for a variety of oral diseases such as oral cancer [2] and the widespread common oral inflammatory disease periodontitis (PD) [3]. The deleterious effects of smoking on tissue integrity are driven by a variety of biological mechanisms. In the recent past, evidence grew that some of these mechanisms correlate with changes in DNA methylation patterns. DNA methylation plays a particular role in cell differentiation and the maintenance of cell specificity [4] and represents an important mechanism for cells to react on persistent external stimuli, allowing somatic cells to adjust gene expression to a particular environment in a long-term manner that can be passed on to daughter cells [5, 6]. Smoking is currently the best studied environmental factor altering DNA-methylation patterns [7]. A plethora of studies exist that show significantly different methylation patterns in smokers compared to nonsmokers in different tissues [2, 8-22] and in the context of smoke-related diseases like cancer [23] or chronic obstructive pulmonary disease (COPD) [21]. The bestreplicated gene-specific association of differential methylation with smoking is attributed to the aryl-hydrocarbon receptor repressor $A H R R$ across various tissues and cell types $[8,10,12-14,16,17,20,22,24,25]$. AHRR is a core regulator in the aryl hydrocarbon receptor (AhR) signalling cascade, modulating dioxin toxicity pathways involving Cytochrome P450, most likely in a cell-specific manner [26]. Most EWAS that assessed the role of CpG methylation in tobacco-related xenobiotic metabolism analysed leukocytes, but some also analysed airway epithelial cells [27] and buccal cells [12, 21, 22]. These cell types are located at the interface to the environment and form a mechanical and physiological barrier. Most notably, a large comprehensive EWAS analysed buccal cells of 790 healthy individuals and identified a set of $1501 \mathrm{CpGs}$ that were differentially methylated in smokers [22]. Buccal swabs largely consist of non-keratinized squamous epithelial cells detached from the inner facial cheeks, and immune cells from the saliva. In the current study, we chose to analyse the effects of cigarette smoke on the healthy keratinized oral mucosa, which is restricted to the gingiva, the tongue and the hard palate. It is the tissue that forms the mechanical barrier between the oral cavity and the hard tissues (alveolar bone and skull). It consists of an upper layer of highly keratinized stratified squamous epithelium, the underlying keratinized connective tissue and invaded immune cells, which make it a strong mechanical and immunological barrier against injuries and pathogens. In addition, the gingiva is the only interface of the human body that is penetrated by hard tissues (the teeth), forming a direct connection between the environment and the bone. In this context, the masticatory mucosa is unique. Accordingly, the gingiva is the tissue which is affected by the common inflammatory disease periodontitis, for which smoking is the strongest risk factor. In contrast, buccal cells are not involved in the pathophysiology of periodontitis. The different functions of the lining mucosa of the inner cheeks, where buccal cells are derived, and of the masticatory mucosa are characterised by pronounced differences in cell turnover [28], cellular differentiation [29], and differences in expression patterns and permeability $[29,30]$. Because of these special features in cell derivation, function and clinical significance, the objective of our study was to assess the putative differences of methylation and transcription of the oral masticatory mucosa between healthy smokers and never smokers. Knowledge of these differences will provide information on the regulatory responses of this interface to direct exposure to xenobiotics of tobacco smoke before periodontitis or oral cancer becomes a clinical manifestation, and if defective, may have a relevant role in the disease aetiology.

To this end, we performed an EWAS with 39 healthy participants, all of them showing no signs of oral inflammation as indicated by a periodontal screening index $(\mathrm{PSI})<2$. Taking into account that the masticatory mucosa itself also differs in cell type composition across different oral regions [31], samples were extracted from a clearly defined site. In this very homogeneous set of ex vivo biopsies, methylation patterns were investigated using the Infinium Methylation EPICBeadChip. In order to investigate potential effects of differentially methylated $\mathrm{CpG}$ sites on gene expression, we additionally performed RNA-sequencing (RNA-Seq) in the same samples. With this study, we provide a genome-wide methylation reference set and a RNA-Sequencing data set for the healthy masticatory mucosa, the clinically affected tissue in periodontitis. For the first time, smoking-informed reference datasets for methylation and expression patterns are combined for the oral mucosa. With these datasets, we were able to validate associations of CpGs with smoking levels shown in buccal swabs [22] and add to the knowledge of potential tissuespecific differential methylation in smokers. We showed shifts in transcriptional patterns of smokers, which partly paralleled the differential methylation patterns.

\section{Results}

DNA methylation differences of the masticatory mucosa between smokers and non-smokers

The DNA of 18 healthy smokers and 21 healthy nonsmokers of the same age was analyzed with the Infinium 
DNA MethylationEPIC BeadChip (Table 1). Next, 802, 254 DNA methylation probes that passed the QC criteria were investigated for statistical significant differences in methylation levels between smokers and never smokers in an ANCOVA adjusting for age, sex and batch effects. A QQ plot revealed some global inflation of the test statistics as compared to the expected distribution, with an inflation factor of $\lambda=1.12$ (Fig. 1a). After correction for multiple testing, we found nine CpGs to be significantly hypomethylated in smokers, with cg04066994 in the AHRR (aryl-hydrogen receptor repressor) gene showing the strongest association $(p=$ $5.9 \times 10^{-10}, \Delta \beta=-0.08$ in smokers; Fig. 1b, Table 2). The overall effect sizes of differentially methylated loci in smokers are illustrated in Fig. 2. Additionally, two significant $\mathrm{CpGs}$ mapped to the intronic region of CYP1B1 and another one to an intergenic region $24 \mathrm{~kb}$ upstream of $C Y P 1 B 1$, three mapped to the long noncoding RNA LINC00673 and one was located in an intergenic region $\sim 6 \mathrm{~kb}$ upstream of CYP1A2 (cytochrome P450 family 1 subfamily A member 2). Observed effect sizes were highest in CYP1B1, with $\Delta \beta=-0.18$ for $\operatorname{cg} 02162897$ $\left(p=5.5 \times 10^{-8}\right)$.

Subsequently, the methylation levels were analysed for association with smoke pack-years (SPY), a measure for smoking intensity that includes information of the quantity of smoking. After correction for multiple testing, 54 CpGs showed significant hypomethylation and seven CpGs showed significant hypermethylation with increasing smoking levels, measured as SPY $(q<0.05$; Figs. 3 and 4 and Table 3$)$. The most significant differential methylation was observed in the 3' untranslated region (3'UTR) of the gene CYP1B1 (cytochrome P450 family 1 subfamily $B$ member 1), with an average change in methylation of $\Delta \beta=-0.046$ per ten SPY at $\operatorname{cg} 01584760\left(p=4.0 \times 10^{-10}\right.$; $\left.q=3.2 \times 10^{-4}\right)$. Additionally, four other CpGs in the intronic region of CYP1B1 showed significant hypomethylation with increasing SPY (e.g. cg20408276, $\Delta \beta=-0.108$ per ten SPY, $\left.p=1.1 \times 10^{-7}\right)$. Another three significant probes $(q<0.05)$ flanked the long noncoding antisense RNA CYP1B1-AS1, which is partially overlapping with CYP1B1 (e.g. cg10090109, $\Delta \beta=-0.015$ per ten SPY, $p=$ $\left.2.1 \times 10^{-8}\right)$. Among the other significant probes $(q<0.05)$, six showed similar or higher effect sizes compared to those observed at CYP1B1. These mapped to the genes

Table 1 Basic characteristics of the EWAS study population

\begin{tabular}{lll}
\hline & Smokers $(n=18)$ & Never smokers $(n=21)$ \\
\hline Sex (males) & $10(55.6 \%)$ & $8(38.1 \%)$ \\
Age, years (mean) & $38 \pm 11$ & $35 \pm 11$ \\
SPY in smokers (mean) & $12 \pm 11$ & NA \\
SPY $\geq 10$ & $9(50 \%)$ & NA \\
SPY 2 $>10$ & $9(50 \%)$ & NA \\
\hline
\end{tabular}

PIP4K2A (phosphatidylinositol-5-phosphate 4-kinase type 2 alpha; cg02030592, $\Delta \beta=-0.106$ per ten SPY, $p=5.3 \times$ $10^{-7}$ ), DLX5 (distal-less homeobox 5; e.g. cg11891395, $\Delta \beta=-0.097$ per ten SPY, $p=2.5 \times 10^{-6}$ ), FABP4 (fatty acid binding protein $4 ; \operatorname{cg} 07234508, \Delta \beta=-0.073$ per ten SPY, $p=1.1 \times 10^{-6}$ ), FABP5P3 (fatty acid binding protein 5 pseudogene 3; $\operatorname{cg} 16171205, \Delta \beta=-0.047$ per ten SPY, $p=$ $\left.6.4 \times 10^{-7}\right)$ and C10orf46 (cg27009448, $\Delta \beta=-0.046$ per ten SPY, $p=3.0 \times 10^{-6}$ ). To account for our small sample size, which was very susceptible to false negative findings, we relaxed the significance threshold to $q<0.15$ and compared the results of the SPY analysis to previously published results from a well-powered EWAS on smoking in buccal cells [22] and to a set of CpGs that was reported to be associated with smoking by multiple independent EWAS performed in blood [32]. Out of $352 \mathrm{CpGs}$ that passed the lowered significance threshold in our data, 33 mapped to 18 loci that were associated in buccal cells, three of which were also reported in blood, among them AHRR (Table 4, Additional file 1). Moreover, two CpGs were associated with $q<0.15$ that mapped to loci associated in blood, but not in buccal cells.

\section{Estimation of different cell type fractions in the masticatory mucosa}

The masticatory mucosa consists of a layer of keratinized epithelium, an underlying layer of connective tissue and invaded immune cells. We calculated the fraction of different cell types with the EpiDISH algorithm. The number of fibroblasts and immune cells in our samples was moderate (median 0.15 and 0.16 , respectively) compared to epithelial cells (median 0.69; Fig. 5a, Additional file 2). The amount of epithelial cells in smokers and never smokers was highly similar (median 0.69 and 0.68 , respectively). Similarly, we observed no differences in the estimated fractions of fibroblasts and immune cells, which seemed to be very slightly reduced in smokers (median of 0.15 and 0.14 , respectively, compared to 0.16 for both cell types in never smokers).

\section{Differential gene expression between smokers and never smokers in the masticatory mucosa}

We performed RNA-Seq of the same samples that were used in the EWAS to quantify the gene expression in the masticatory mucosa. Among the ten highest expressed genes in the solid oral mucosa, six were keratin genes (KRT1, KRT6A, KRT10, KRT14, KRT16 and KRT76 with median TPM values between 118,399 and 355,686; Additional file 3). The other genes of the top ten highest expressed genes were $S 100 A 8$ and $5100 A 9$ of the S100 protein family, which has a prominent role in the regulation of inflammatory processes and immune response, COX1 (cytochrome c oxidase subunit 1) and EEF1A1 (elongation factor 1-alpha 1). 

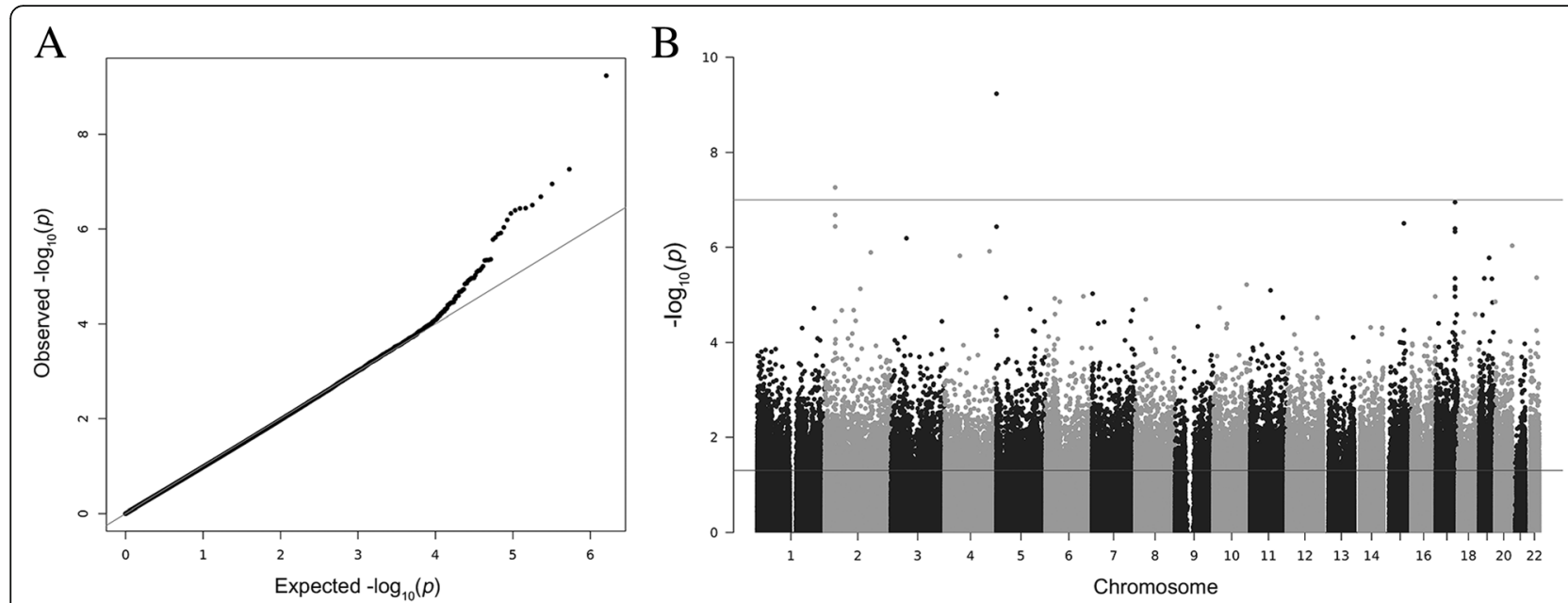

Fig. 1 Manhattan and QQ plots for epigenome-wide associations with smoking status in the masticatory mucosa. a The QQ plot showed some evidence of inflated association signals $\left(\lambda=1.12\right.$ ). $\mathbf{b}$ Manhattan plot showing - $\log _{10}$ transformed $p$ values from the ANCOVA plotted against the genomic location of the probes. The horizontal lines indicate the nominal significance threshold $(p<0.05)$ and the genome-wide significance threshold $\left(p<10^{-7}\right)$

After adjustment for multiple testing according to Benjamini \& Hochberg, 20 genes showed differential expression between smokers and never smokers (Table 5). The most significant association of differential expression and smoking status was found for CYP1B1 $\left(p=2.2 \times 10^{-14}, q=4.9 \times\right.$ $10^{-10}, \log 2$ fold change $=2.9$ ). A KEGG pathway analysis showed a significant upregulation of two pathways, the Metabolism of Xenobiotics by Cytochrome P450 Pathway, (KEGG entry: rn00980; $p=3.7 \times 10^{-4} ; q=0.034$; Table 6) and the Aminoacyl-tRNA biosynthesis pathway (KEGG entry: hsa00970; $\left.p=1.9 \times 10^{-4} ; q=0.034\right)$. A Reactome pathway analysis showed 24 pathways to be significantly upregulated in smokers $(q<0.05)$. Out of these, 14 pathways belonged to the pathway classes of "cell cycle function" and/or "DNA replication" and three to the pathway class "metabolism" with respect to electron transport, most notably the top significant pathway involving the Citric Acid Cycle and Respiratory Electron Transport (Reactome identifier: R-HSA-1428517; $p=1.4 \times 10^{-5} ; q=0.009$ ). In both approaches, no significantly downregulated pathways were found (Additional file 4).

We next investigated whether changes in methylation patterns have a measurable effect on gene expression in vivo. At a significance threshold of $\mathrm{FDR}=0.05, C Y P 1 B 1$ was the only gene that showed correlation of hypomethylation with gene expression. Considering the limited statistical power of our sample that made it susceptible for false negative associations, we relaxed the significant

Table 2 Change in DNA methylation $\beta$ values for smokers compared to never smokers

\begin{tabular}{|c|c|c|c|c|c|c|c|}
\hline CpG identifier & Chr & Position in bp (hg19 $)$ & Gene symbol & Region & $\Delta \beta$ & $p$ value $^{b}$ & $q$ value \\
\hline cg02162897 & 2 & $38,300,537$ & $C Y P 1 B 1^{\complement}$ & Body & -0.183 & $5.5 \times 10^{-8}$ & 0.022 \\
\hline cg20408276 & 2 & $38,300,586$ & $C Y P 1 B 1^{\mathrm{C}}$ & Body & -0.176 & $3.6 \times 10^{-7}$ & 0.040 \\
\hline cg24752487 & 2 & $38,324,188$ & $21 \mathrm{~kb}$ upstream of $C Y P 1 B 1^{c}$ & $\begin{array}{l}\text { Intergenic; Mammalian H3K27me3- } \\
\text { repression mark }\end{array}$ & -0.138 & $2.1 \times 10^{-7}$ & 0.040 \\
\hline cg06036945 & 5 & 394,869 & $A H R R^{C}$ & Body & -0.125 & $3.7 \times 10^{-7}$ & 0.040 \\
\hline cg04066994 & 5 & 394,891 & $A H R R^{C}$ & Body & -0.083 & $5.9 \times 10^{-10}$ & $4.7 \times 10^{-2}$ \\
\hline cg20385913 & 15 & $75,054,510$ & $6 \mathrm{~kb}$ downstream of CYP1A2 & intergenic & -0.063 & $3.1 \times 10^{-7}$ & 0.040 \\
\hline $\operatorname{cg} 02123605$ & 17 & $70,497,550$ & LINC00673 ${ }^{C}$ & Body & -0.056 & $4.0 \times 10^{-7}$ & 0.040 \\
\hline $\operatorname{cg} 23167235$ & 17 & $70,532,667$ & LINC00673C & Body & -0.018 & $4.7 \times 10^{-7}$ & 0.042 \\
\hline $\operatorname{cg} 23398508$ & 17 & $70,536,199$ & $\operatorname{LINC00673}^{\mathrm{C}}$ & Body & -0.098 & $1.1 \times 10^{-7}$ & 0.030 \\
\hline
\end{tabular}

Shown are results for all CpGs significant after correction for multiple testing $(q<0.05)$. For comparison, we additionally included the results from the SPY analysis for significant $\mathrm{CpGs}$

${ }^{a} \mathrm{NCBI}$ build GRCh37

${ }^{b} p$ values are from the analysis of covariance (ANCOVA) with adjustment by age, sex and batch. Chr chromosome, bp basepairs, SPY smoke pack years, $k b$ kilobasepairs

'Genetic region previously reported to be associated with smoking in buccals [22] 


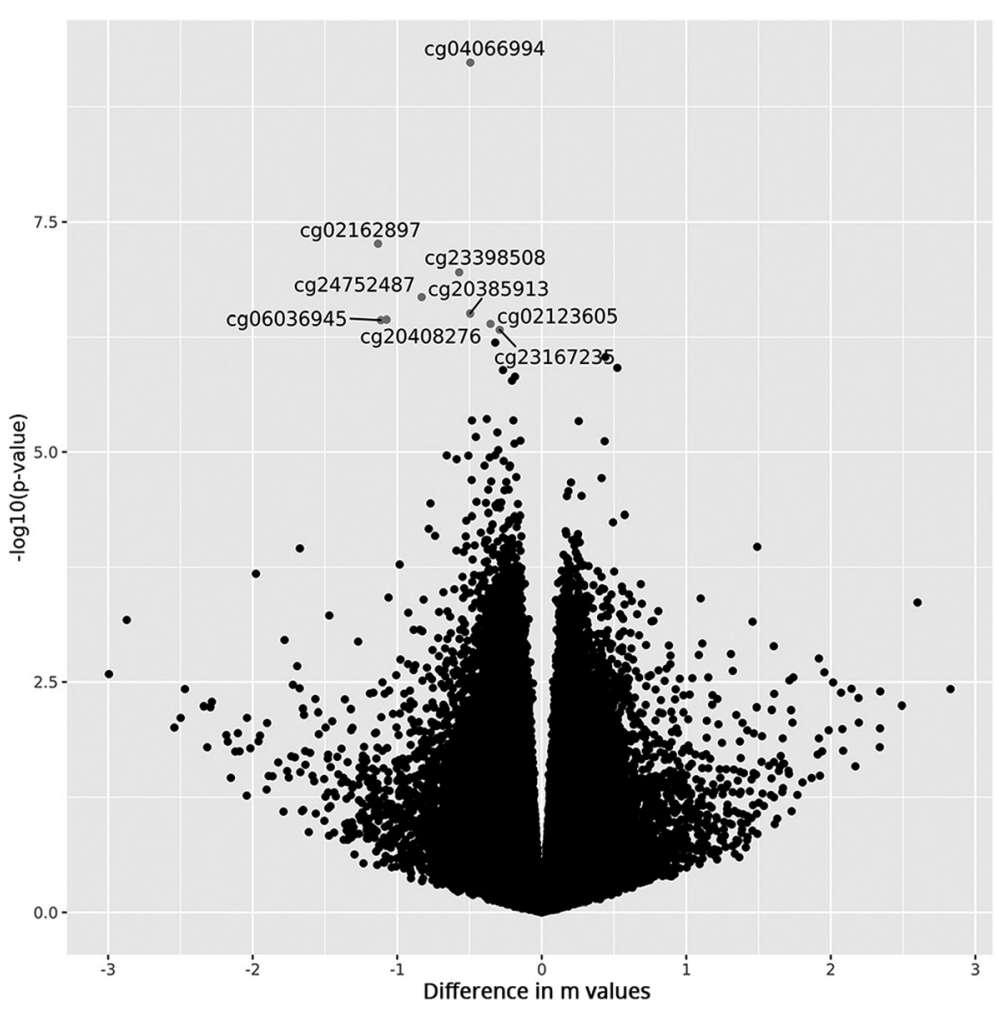

Fig. 2 Volcano plot showing methylation differences of smokers compared to never smokers against -log 10 of $p$ values from the ANCOVA. Identifiers are given for the CpGs that were significantly associated with smoking after adjustment for multiple testing

thresholds and correlated all CpGs with $q<0.15$ to those genes that were differentially expressed in smokers with a nominal $p$ value of $<0.05$. This analysis suggested differential expression of 15 genes with 25 CpGs at $q<0.15$ (Additional file 5). To balance the resulting increased risk of type I errors, we subsequently included only those genes that mapped to at least two associated CpGs $(q<0.15)$ in our EWAS and had previously been reported to be associated with smoking in buccal swabs or blood [22, 32]. In total, four genes fulfilled these criteria:
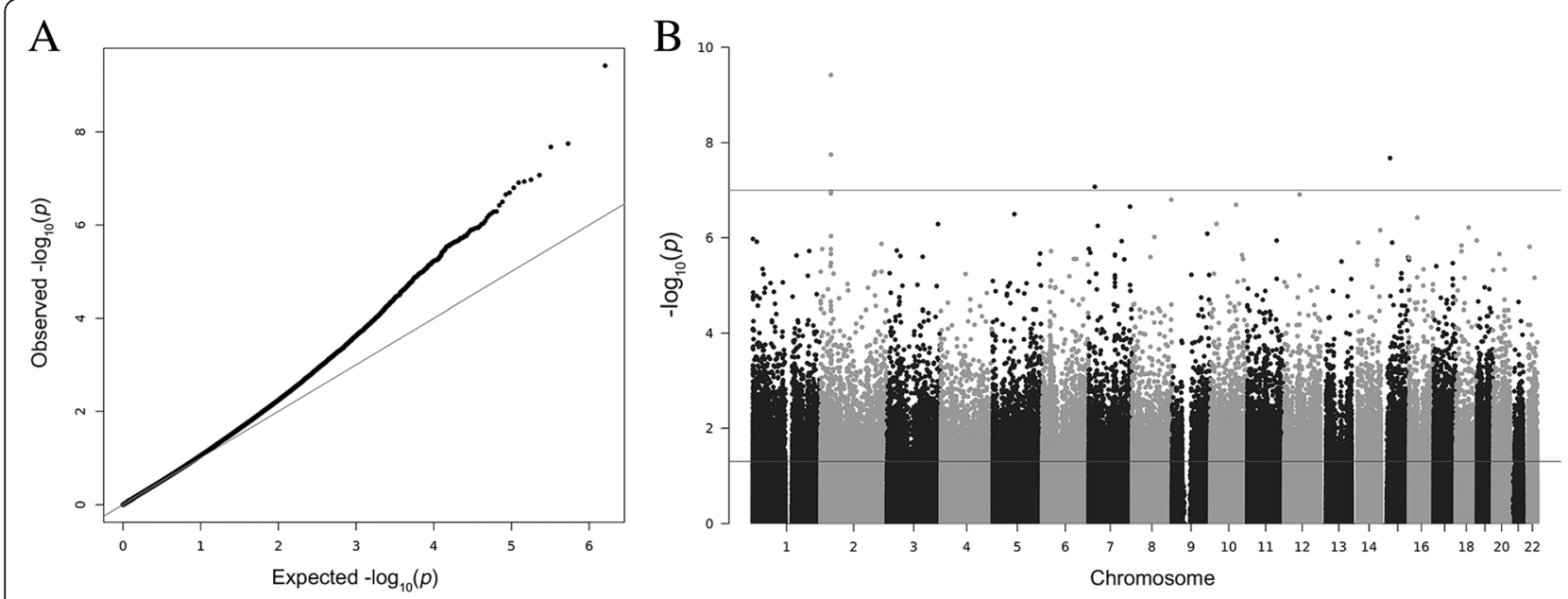

Fig. 3 Manhattan and QQ pots for epigenome-wide associations with SPY in the masticatory mucosa. a The QQ plot showed some evidence for inflation of association signals $(\lambda=1.09)$. b Manhattan plot showing $\log _{10}$ transformed $p$ values from the ANCOVA plotted against the genomic location of the probes. The horizontal lines indicate the nominal significance threshold $(p<0.05)$ and the genome-wide significance threshold $\left(p<10^{-7}\right)$ 


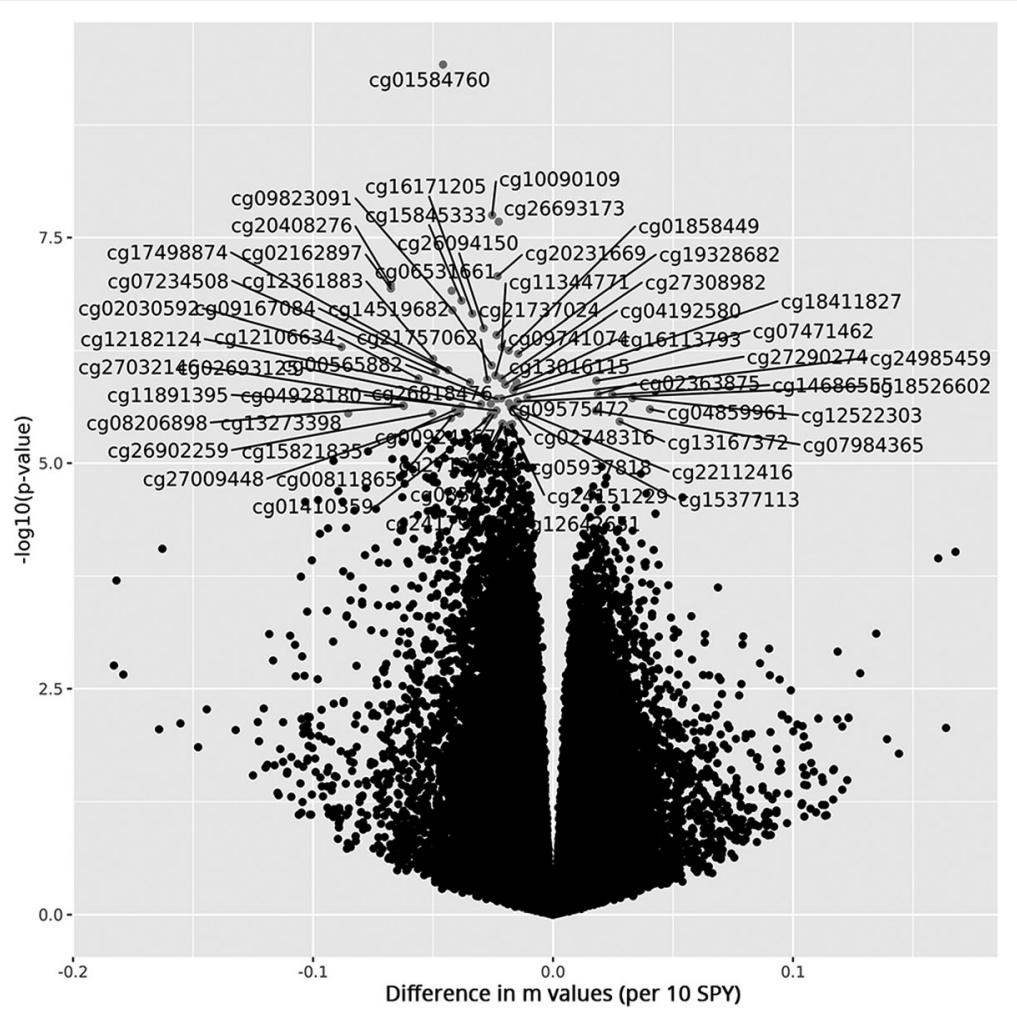

Fig. 4 Volcano plot showing methylation differences per 10 SPY against - $\log 10$ of $p$ values from the ANCOVA. Identifiers are given for the CpGs that were significantly associated with an increase in smoking after adjustment for multiple testing

CYP1B1, SLC7A5 (solute carrier family 7 member 5), EDC3 (enhancer of mRNA decapping 3) and LAMA3 (laminin subunit alpha 3), the latter of which showed opposite effect sizes for differential expression and differential methylation (Table 7). Because the differentially methylated lncRNA CYP1B1-AS1 was not covered by our RNA-Seq reads, we analysed the expression by qRT-PCR in 69 individuals $(n=32$ smokers, 37 never smokers). CYP1B1-AS1 was stronger expressed in smokers when compared to never smokers with $p=0.0061$.

In vitro effects of cigarette smoke extract on the expression of hypomethylated genes in primary gingival fibroblasts

CYP1B1 had been shown to be induced in response to cigarette smoke exposure in the adenocarcinomic human alveolar basal epithelial cell line A549 from cancerous lung tissue [23]. To validate the regulatory effect of tobacco smoke on $C Y P 1 B 1$ gene expression for gingival cells, we exposed gingival fibroblasts to cigarette smoke extract (CSE) in vitro. Exposure to CSE significantly increased the mRNA levels of CYP1B1 and of the corresponding antisense RNA CYP1B1-AS with $p=0.019$ and $p=0.023$, respectively (Fig. 6).
Association of smoking-induced changes in methylation levels in oral squamous cell carcinoma

To investigate a potential role of smoking-induced CYP1B1 and AHRR hypomethylation in the development of oral cancer, we compared the methylation data from our healthy never smokers with the TCGA data for 16 oral squamous cell carcinoma (OSCC) biopsies that were specified as "gum" or "palate", similar to the samples used in our EWAS. As the exact extraction site was not specified for OSCC samples, we first investigated cell type compositions using EpiDISH in this potentially heterogeneous tissue source. The cell composition varied extremely among OSCC samples, irrespective of smoking status (Fig. 5b, Additional file 2), with an overall higher amount of epithelial cells compared to our EWAS never smokers (median of 0.80 in OSCC compared to 0.69 in our samples). The OSCC data are based on the $450 \mathrm{k}$ array, and of a total of $202 \mathrm{CpGs}$ at the genetic region of AHRR and CYP1B1 that were analysed in our EPIC array dataset, $36 \%(n=72)$ were not analysed with the $450 \mathrm{k}$ array. Yet, a total of $130 \mathrm{CpGs}$ at $A H R R$ ( $n=98$ CpGs) and CYP1B1 ( $n=32 \mathrm{CpGs})$ were shared between the OSCC samples and our data. In the OSCC data, at CYP1B1, 1 CpG was significantly hypomethylated and 16 CpG were significantly hypermethylated (corrected 
Table 3 Change in DNA methylation $\beta$ values per ten smoking pack years for significant CpGs

\begin{tabular}{|c|c|c|c|c|c|c|c|}
\hline CpG identifier & Chr & Position in bp (hg19a) & Gene symbol & Region & $\Delta \beta$ per $10 \mathrm{SPY}$ & $p$ value ${ }^{b}$ & $q$ value \\
\hline cg09741074 & 1 & $1,310,496$ & AURKAIP1 & 5'UTR, 1st Exon & -0.002 & $8.2 \times 10^{-7}$ & $3.5 \times 10^{-2}$ \\
\hline $\operatorname{cg} 27290274$ & 1 & $15,386,739$ & KIAA1026 & Body & 0.003 & $1.2 \times 10^{-6}$ & $3.9 \times 10^{-2}$ \\
\hline $\operatorname{cg} 22112416$ & 1 & $161,124,001$ & UFC1 & Body & -0.003 & $2.7 \times 10^{-6}$ & $4.1 \times 10^{-2}$ \\
\hline cg12522303 & 1 & $207,818,368$ & CR1L & TSS200 & 0.009 & $2.8 \times 10^{-6}$ & $4.2 \times 10^{-2}$ \\
\hline $\operatorname{cg} 14686555$ & 2 & $6,121,915$ & LOC400940 & TSS200 & 0.005 & $2.1 \times 10^{-6}$ & $4.1 \times 10^{-2}$ \\
\hline cg08502446 & 2 & $36,891,478$ & & Intergenic & -0.017 & $3.5 \times 10^{-6}$ & $4.6 \times 10^{-2}$ \\
\hline cg01584760 & 2 & $38,296,474$ & $C Y P 1 B 1^{c, d}$ & $3^{\prime} \mathrm{UTR}$ & -0.046 & $4.0 \times 10^{-10}$ & $3.2 \times 10^{-4}$ \\
\hline cg02162897 & 2 & $38,300,537$ & $C Y P 1 B 1^{c, d}$ & Body & -0.110 & $1.1 \times 10^{-7}$ & $1.3 \times 10^{-2}$ \\
\hline cg20408276 & 2 & $38,300,586$ & $C Y P 1 B 1^{c, d}$ & Body & -0.108 & $1.1 \times 10^{-7}$ & $1.3 \times 10^{-2}$ \\
\hline cg00565882 & 2 & $38,300,707$ & $C Y P 1 B 1^{c, d}$ & Body & -0.041 & $1.7 \times 10^{-6}$ & $4.1 \times 10^{-2}$ \\
\hline cg01410359 & 2 & $38,302,230$ & $C Y P 1 B 1^{c, d}$ & Body & -0.037 & $3.6 \times 10^{-6}$ & $4.6 \times 10^{-2}$ \\
\hline $\operatorname{cg} 26818476$ & 2 & $38,337,733$ & CYPIBI-AS & Upstream & -0.021 & $2.3 \times 10^{-6}$ & $4.1 \times 10^{-2}$ \\
\hline $\operatorname{cg} 10090109$ & 2 & $38,338,120$ & CYPIBI-AS & Upstream & -0.015 & $2.1 \times 10^{-8}$ & $5.9 \times 10^{-3}$ \\
\hline cg17498874 & 2 & $38,460,564$ & CYPIBI-AS & Downstream & -0.062 & $1.1 \times 10^{-6}$ & $3.9 \times 10^{-2}$ \\
\hline cg04192580 & 2 & $223,726,348$ & ACSL3 & 5'UTR & -0.008 & $1.3 \times 10^{-6}$ & $4.0 \times 10^{-2}$ \\
\hline $\operatorname{cg} 24985459$ & 3 & $37,035,090$ & MLHI & TSS200, 1st Exon & -0.001 & $2.5 \times 10^{-6}$ & $4.1 \times 10^{-2}$ \\
\hline cg02748316 & 3 & $50,273,710$ & GNAI2 & 5'UTR & -0.002 & $2.5 \times 10^{-6}$ & $4.1 \times 10^{-2}$ \\
\hline cg04859961 & 3 & $131,951,282$ & CPNE4; tRNA $A^{C y s}$ & Body & 0.024 & $2.6 \times 10^{-6}$ & $4.1 \times 10^{-2}$ \\
\hline $\operatorname{cg} 11344771$ & 3 & $188,108,786$ & $\angle P P$ & 5'UTR & -0.020 & $6.5 \times 10^{-7}$ & $3.1 \times 10^{-2}$ \\
\hline cg26094150 & 5 & $79,378,871$ & THBS4 & Body & -0.003 & $2.9 \times 10^{-7}$ & $2.2 \times 10^{-2}$ \\
\hline cg24179483 & 5 & $170,814,656$ & NPM1 & TSS200 & -0.002 & $3.1 \times 10^{-6}$ & $4.3 \times 10^{-2}$ \\
\hline cg09575472 & 5 & $174,045,109$ & & Intergenic & -0.032 & $2.0 \times 10^{-6}$ & $4.1 \times 10^{-2}$ \\
\hline cg07471462 & 6 & $33,168,624$ & SLC39A7 & 1stExon, 5'UTR & -0.002 & $1.8 \times 10^{-6}$ & $4.1 \times 10^{-2}$ \\
\hline $\operatorname{cg} 13273398$ & 6 & $116,937,346$ & RSPH4A & TSS1500 & -0.018 & $2.7 \times 10^{-6}$ & $4.1 \times 10^{-2}$ \\
\hline cg26902259 & 6 & $126,102,246$ & NCOA7 & TSS200 & -0.008 & $2.6 \times 10^{-6}$ & $4.1 \times 10^{-2}$ \\
\hline cg02363875 & 7 & 960,707 & ADAP1 & TSS200 & 0.003 & $1.7 \times 10^{-6}$ & $4.1 \times 10^{-2}$ \\
\hline cg18526602 & 7 & $6,487,739$ & $D A G L B$ & TSS200 & -0.002 & $2.0 \times 10^{-6}$ & $4.1 \times 10^{-2}$ \\
\hline cg20231669 & 7 & $22,862,119$ & TOMM7 & Body & -0.005 & $9.2 \times 10^{-8}$ & $1.3 \times 10^{-2}$ \\
\hline cg01858449 & 7 & $33,551,455$ & BBS9 & Body & -0.020 & $6.2 \times 10^{-7}$ & $3.1 \times 10^{-2}$ \\
\hline cg27032146 & 7 & $96,652,115$ & $D L X 5^{c}$ & Body & -0.062 & $2.4 \times 10^{-6}$ & $4.1 \times 10^{-2}$ \\
\hline cg11891395 & 7 & $96,652,153$ & $D L X 5^{C}$ & Body & -0.097 & $2.5 \times 10^{-6}$ & $4.1 \times 10^{-2}$ \\
\hline $\operatorname{cg} 21757062$ & 7 & $121,376,168$ & $329 \mathrm{bp}$ from $75 \mathrm{~K}$ & Intergenic & -0.027 & $1.5 \times 10^{-6}$ & $4.1 \times 10^{-2}$ \\
\hline cg00924357 & 7 & $131,012,552$ & MKLN1 & Body & -0.001 & $2.6 \times 10^{-6}$ & $4.1 \times 10^{-2}$ \\
\hline $\operatorname{cg} 16171205$ & 7 & $152,135,221$ & FABP5P3 & Body & -0.047 & $3.0 \times 10^{-7}$ & $2.2 \times 10^{-2}$ \\
\hline cg15821835 & 8 & $67,686,767$ & SGK3 & TSS1500 & -0.006 & $2.3 \times 10^{-6}$ & $4.1 \times 10^{-2}$ \\
\hline cg07234508 & 8 & $82,395,948$ & FABP4 & TSS1500 & -0.073 & $1.1 \times 10^{-6}$ & $3.9 \times 10^{-2}$ \\
\hline $\operatorname{cg} 15845333$ & 8 & $144,417,025$ & TOP1MT & 1stExon & -0.003 & $1.6 \times 10^{-7}$ & $1.6 \times 10^{-2}$ \\
\hline cg14519682 & 9 & $130,213,309$ & LRSAM1 & TSS1500 & -0.002 & $8.0 \times 10^{-7}$ & $3.5 \times 10^{-2}$ \\
\hline cg02030592 & 10 & $22,863,044$ & PIP4K2A & Body & -0.106 & $5.3 \times 10^{-7}$ & $3.1 \times 10^{-2}$ \\
\hline cg09823091 & 10 & $94,644,717$ & EXOC6 & Body & -0.033 & $1.8 \times 10^{-7}$ & $1.6 \times 10^{-2}$ \\
\hline cg08206898 & 10 & $116,581,183$ & FAM160B1 & TSS1500 & -0.016 & $2.2 \times 10^{-6}$ & $4.1 \times 10^{-2}$ \\
\hline $\operatorname{cg} 27009448$ & 10 & $120,492,138$ & C10orf46 & Body & -0.046 & $3.0 \times 10^{-6}$ & $4.3 \times 10^{-2}$ \\
\hline $\operatorname{cg} 12106634$ & 11 & $108,092,400$ & ATM & TSS1500 & -0.006 & $1.2 \times 10^{-6}$ & $3.9 \times 10^{-2}$ \\
\hline cg06531661 & 12 & $57,522,120$ & LRP1 & TSS200 & -0.002 & $1.1 \times 10^{-7}$ & $1.3 \times 10^{-2}$ \\
\hline
\end{tabular}


Table 3 Change in DNA methylation $\beta$ values per ten smoking pack years for significant CpGs (Continued)

\begin{tabular}{|c|c|c|c|c|c|c|c|}
\hline CpG identifier & Chr & Position in bp (hg19a) & Gene symbol & Region & $\Delta \beta$ per 10 SPY & $p$ value $^{\mathrm{b}}$ & $q$ value \\
\hline $\operatorname{cg} 13016115$ & 14 & $23,388,406$ & RBM23 & TSS200 & -0.002 & $1.3 \times 10^{-6}$ & $4.0 \times 10^{-2}$ \\
\hline cg24151229 & 14 & $93,260,858$ & GOLGA5 & $5^{\prime} \cup T R$ & -0.002 & $3.6 \times 10^{-6}$ & $4.6 \times 10^{-2}$ \\
\hline $\operatorname{cg} 15377113$ & 14 & $93,799,418$ & BTBDX & TSS200 & -0.001 & $3.1 \times 10^{-6}$ & $4.3 \times 10^{-2}$ \\
\hline $\operatorname{cg} 12361883$ & 14 & $104,313,596$ & LINC00637 & TSS1500 & -0.002 & $6.4 \times 10^{-7}$ & $3.1 \times 10^{-2}$ \\
\hline $\operatorname{cg} 26693173$ & 15 & $33,137,114$ & FMN1 & Body & -0.015 & $2.2 \times 10^{-8}$ & $5.9 \times 10^{-3}$ \\
\hline cg09167084 & 15 & $40,861,240$ & RPUSD2 & TSS1500 & -0.008 & $1.1 \times 10^{-6}$ & $3.9 \times 10^{-2}$ \\
\hline cg04928180 & 15 & $99,791,525$ & $\angle R R C 28$ & TSS200 & -0.001 & $2.3 \times 10^{-6}$ & $4.1 \times 10^{-2}$ \\
\hline cg07984365 & 16 & 739,425 & WDR24 & 1stExon & 0.011 & $2.5 \times 10^{-6}$ & $4.1 \times 10^{-2}$ \\
\hline cg21737024 & 16 & $30,441,289$ & DCTPP1 & 1stExon & -0.003 & $3.8 \times 10^{-7}$ & $2.6 \times 10^{-2}$ \\
\hline $\operatorname{cg} 12642651$ & 17 & $9,861,712$ & GAS7 & Body & -0.022 & $3.6 \times 10^{-6}$ & $4.6 \times 10^{-2}$ \\
\hline $\operatorname{cg} 13167372$ & 17 & $70,536,814$ & LINC00673 $3^{\mathrm{c}, \mathrm{d}}$ & Gene body & 0.027 & $2.2 \times 10^{-6}$ & $4.1 \times 10^{-2}$ \\
\hline $\operatorname{cg} 12182124$ & 18 & $21,451,563$ & $\angle A M A 3^{C}$ & TSS1500 & -0.040 & $2.2 \times 10^{-6}$ & $4.1 \times 10^{-2}$ \\
\hline cg16113793 & 18 & $21,451,607$ & $\angle A M A 3^{C}$ & TSS1500 & -0.028 & $1.8 \times 10^{-6}$ & $4.1 \times 10^{-2}$ \\
\hline cg19328682 & 18 & $47,807,700$ & MBD1 & $5^{\prime}$ UTR & -0.004 & $6.1 \times 10^{-7}$ & $3.1 \times 10^{-2}$ \\
\hline cg27308982 & 18 & $77,196,450$ & NFATC1 & Body, 5'UTR & -0.012 & $1.1 \times 10^{-6}$ & $3.9 \times 10^{-2}$ \\
\hline cg02693125 & 20 & $23,401,585$ & $N A P B$ & Body & -0.006 & $1.9 \times 10^{-6}$ & $4.1 \times 10^{-2}$ \\
\hline $\operatorname{cg} 18411827$ & 22 & $23,412,613$ & GNAZ & TSS200 & -0.003 & $1.4 \times 10^{-6}$ & $4.0 \times 10^{-2}$ \\
\hline
\end{tabular}

Shown are results for all CpGs significant after correction for multiple testing $(q<0.05)$

${ }^{\mathrm{a}} \mathrm{NCBI}$ build GRCh37

${ }^{\mathrm{b}} p$ values are from the analysis of covariance (ANCOVA) with SPY as a continuous variable and adjustment by age, sex and batch. Chr chromosome, bp basepairs, SPY smoke pack years

'Genetic region previously reported to be associated with smoking in buccals [22]

${ }^{\mathrm{d}}$ Shared association with the analysis of smoking status

for multiple testing, $n=130$ tests). At AHRR, 30 CpGs were significantly hypomethylated and 22 CpGs were significantly hypermethylated. The effect sizes in OSCC were high, with $\Delta \beta=-0.48$ for $\operatorname{cg} 12202185$ in $A H R R$ $\left(p=2.8 \times 10^{-7}\right)$, and $\Delta \beta=0.45$ for $\operatorname{cg} 09799983$ in CYP1B1 $\left(p=7.5 \times 10^{-12}\right)$, which both were not associated with smoking in our samples (Additional file 6). In our EWAS, five CpGs within CYP1B1 showed significant hypermethylation in smokers, two of which were not analysed on the 450k chip for OSCC. The remaining three were significantly hypermethylated in OSCC, with the highest effect size at cg02162897 with $\Delta \beta=0.197$ $\left(p=4.7 \times 10^{-6}\right.$, Table 8). For AHRR, two CpGs were significantly associated in our EWAS but were not covered by the 450k chip used for the OSCC data. This is why we investigated the CpGs that flanked our associated CpGs in the EWAS, albeit these were not associated in our EWAS. In OSCC, one of these adjacent CpGs, cg04551776, showed a significant hypermethylation $\left(\Delta \beta=0.15, p=2.7 \times 10^{-5}\right)$.

\section{Discussion}

In the current EWAS, we generated a reference dataset for combined differential gene methylation and expression patterns of the healthy masticatory mucosa of never smokers and smokers, accompanied by corresponding estimations of cell type fractions. We identified $63 \mathrm{sig}$ nificant differentially methylated CpGs after adjustment for multiple testing. Of these, the differential methylation at the gene CYP1B1 had the highest effect size, with $11 \%$ hypomethylation per ten SPY. CYP1B1 is a member of the cytochrome $\mathrm{P} 450$ gene family, and the gene product is involved in detoxification of pro-carcinogens in tobacco smoke [33]. CYP1B1 was previously reported to be hypomethylated with epigenome-wide significance in EWAS of buccal swabs [22] and small airway epithelial cells of healthy smokers [27]. The significantly associated genes PIP4K2A and DLX5 showed similar effect sizes as $C Y P 1 B 1$. Interestingly, the GWAS catalogue reports an association with variants within PIP4K2A for several cancer types. For $D L X 5$, which was also found to be significantly associated with SPY in buccal cells [22], the GWAS catalogue reports variants associated with bone mineral densities and facial morphology. There is substantial evidence that smokers suffer from lower bone mineral densities [34, 35], possibly due to increased bone resorption [36]. Smoke-related toxins were described as inducing bone loss mediated by the AhR-pathway involving CYP1A1, CYP1A2 and CYP1B1 in mice [37]. Interestingly, in the analysis of smokers against never smokers as a categorical value, we found nine CpGs to be significantly differentially methylated, with the top 
Table 4 Change in DNA methylation $\beta$ values per ten smoking pack years for validated CpGs from the literature

\begin{tabular}{|c|c|c|c|c|c|c|}
\hline CpG identifier & Chr & Position in bp (hg19a) & Gene symbol & $\Delta \beta$ per 10 SPY & $p$ value $^{\mathrm{b}}$ & $q$ value \\
\hline \multicolumn{7}{|c|}{ Associations in buccals and blood } \\
\hline cg04066994 & 5 & 394,891 & AHRR & -0.04 & $8.2 \times 10^{-6}$ & 0.07 \\
\hline $\operatorname{cg} 14389122^{d}$ & 15 & $74,945,851$ & EDC3 & -0.05 & $1.3 \times 10^{-5}$ & 0.08 \\
\hline $\operatorname{cg} 17181940^{c}$ & 15 & $74,948,075$ & EDC3 & -0.05 & $5.6 \times 10^{-6}$ & 0.06 \\
\hline \multicolumn{7}{|c|}{ Associations in buccals } \\
\hline cg05223638 & 1 & $3,036,168$ & PRDM16 & -0.02 & $5.6 \times 10^{-5}$ & 0.14 \\
\hline cg27290274 & 1 & $15,386,739$ & KIAA1026 & 0.00 & $1.2 \times 10^{-6}$ & 0.04 \\
\hline $\operatorname{cg} 01584760^{c}$ & 2 & $38,296,474$ & CYP1B1 & -0.05 & $3.8 \times 10^{-10}$ & $3.2 \times 10^{-4}$ \\
\hline $\operatorname{cg} 02162897^{d}$ & 2 & $38,300,537$ & CYP1B1 & -0.11 & $1.2 \times 10^{-7}$ & 0.01 \\
\hline $\operatorname{cg} 20408276^{d}$ & 2 & $38,300,586$ & CYP1B1 & -0.11 & $1.1 \times 10^{-7}$ & 0.01 \\
\hline $\operatorname{cg} 00565882^{d}$ & 2 & $38,300,707$ & CYP1B1 & -0.04 & $1.7 \times 10^{-6}$ & 0.04 \\
\hline cg01410359 & 2 & $38,302,230$ & CYP1B1 & -0.04 & $3.4 \times 10^{-6}$ & 0.05 \\
\hline cg03890222 & 2 & $38,302,487$ & CYP1B1 & -0.02 & $6.2 \times 10^{-5}$ & 0.15 \\
\hline $\operatorname{cg} 21715189^{d}$ & 2 & $38,304,802$ & CYP1B1 & -0.06 & $3.2 \times 10^{-5}$ & 0.11 \\
\hline cg06722870 & 2 & $236,840,187$ & AGAP1 & 0.00 & $6.0 \times 10^{-5}$ & 0.14 \\
\hline $\operatorname{cg} 04578109^{c}$ & 4 & $169,475,207$ & PALLD & -0.01 & $1.5 \times 10^{-5}$ & 0.08 \\
\hline cg21490342 & 6 & $31,852,301$ & EHMT2 & 0.01 & $6.5 \times 10^{-5}$ & 0.15 \\
\hline cg23076086 & 6 & $112,194,849$ & FYN & -0.01 & $2.1 \times 10^{-5}$ & 0.09 \\
\hline cg18873386 & 7 & $96,651,915$ & $D L X 5$ & -0.06 & $3.4 \times 10^{-5}$ & 0.11 \\
\hline $\operatorname{cg} 11367354$ & 7 & $96,651,983$ & $D L X 5$ & -0.05 & $1.1 \times 10^{-5}$ & 0.07 \\
\hline $\operatorname{cg} 27032146$ & 7 & $96,652,115$ & DLX5 & -0.06 & $2.4 \times 10^{-6}$ & 0.04 \\
\hline cg11500797 & 7 & $96,652,123$ & $D L X 5$ & -0.07 & $7.1 \times 10^{-6}$ & 0.06 \\
\hline $\operatorname{cg} 11891395$ & 7 & $96,652,153$ & $D L X 5$ & -0.10 & $2.3 \times 10^{-6}$ & 0.04 \\
\hline cg03532926 & 7 & $153,584,839$ & DPP6 & 0.01 & $1.3 \times 10^{-5}$ & 0.08 \\
\hline cg06324554 & 7 & $157,495,841$ & PTPRN2 & -0.06 & $4.9 \times 10^{-5}$ & 0.13 \\
\hline cg02583494 & 9 & $97,640,188$ & C9orf3 & -0.03 & $4.2 \times 10^{-5}$ & 0.12 \\
\hline cg22754866 & 10 & $99,771,121$ & CRTAC1 & -0.03 & $8.5 \times 10^{-6}$ & 0.07 \\
\hline cg15377113 & 14 & $93,799,418$ & BTBD7 & 0.00 & $3.0 \times 10^{-6}$ & 0.04 \\
\hline $\operatorname{cg} 12344004$ & 15 & $89,877,906$ & POLG & -0.01 & $4.1 \times 10^{-5}$ & 0.12 \\
\hline cg10099957 & 16 & $87,869,757$ & SLC7A5 & -0.06 & $9.6 \times 10^{-6}$ & 0.07 \\
\hline $\operatorname{cg} 09285525$ & 16 & $87,869,786$ & SLC7A5 & -0.06 & $1.3 \times 10^{-5}$ & 0.08 \\
\hline $\operatorname{cg} 27555036$ & 16 & $87,898,191$ & SLC7A5 & -0.03 & $4.5 \times 10^{-5}$ & 0.13 \\
\hline $\operatorname{cg} 13167372^{d}$ & 17 & $70,536,814$ & LINC00673 & 0.03 & $3.4 \times 10^{-6}$ & 0.05 \\
\hline $\operatorname{cg} 12182124$ & 18 & $21,451,563$ & LAMA3 & -0.04 & $1.9 \times 10^{-6}$ & 0.04 \\
\hline cg16113793 & 18 & $21,451,607$ & LAMA3 & -0.03 & $1.5 \times 10^{-6}$ & 0.04 \\
\hline \multicolumn{7}{|c|}{ Associations in blood } \\
\hline $\operatorname{cg} 06566772^{c}$ & 10 & $63,661,030$ & ARID5B & 0.00 & $1.7 \times 10^{-5}$ & 0.09 \\
\hline $\operatorname{cg} 16611234^{d}$ & 11 & $58,870,075$ & $\sim 4.5 \mathrm{~kb}$ from FAM111B & -0.03 & $3.9 \times 10^{-5}$ & 0.12 \\
\hline
\end{tabular}

Shown are results for all CpGs that map to genes previously reported in a large EWAS on buccal swabs [22] or in a systematic review on EWAS on blood [32] and which were associated with SPY in our EWAS with $q<0.15$

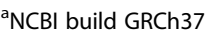

${ }^{\mathrm{b}} p$ values are from the analysis of covariance (ANCOVA) with adjustment by age, sex and batch. Chr chromosome, bp basepairs, kb kilobasepairs, SPY smoke pack years

'Auxiliary probe on EPIC chip

${ }^{\mathrm{d}}$ Same CpG reported in previous EWAS 

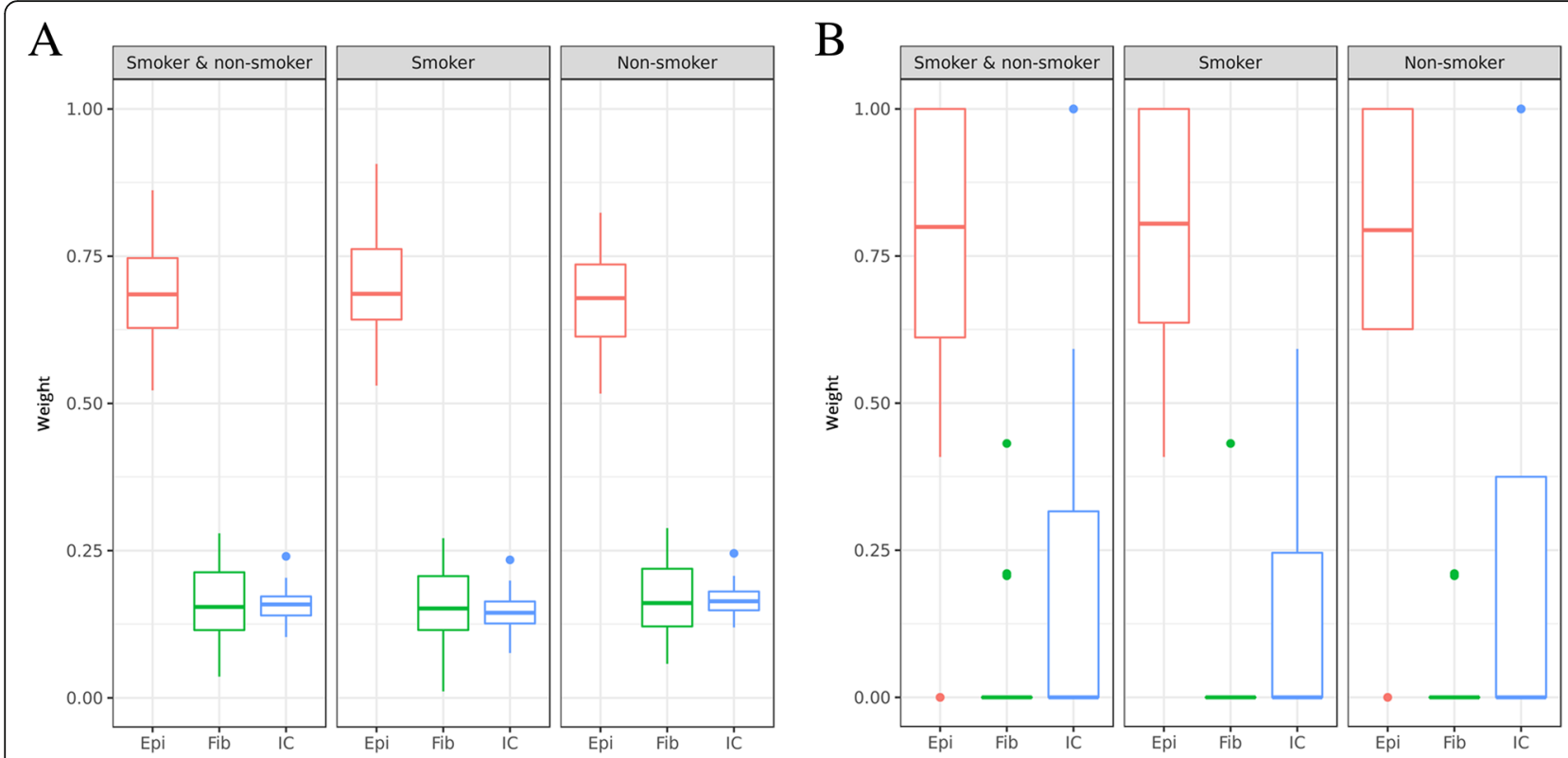

Fig. 5 Cell type estimations in the masticatory mucosa inferred by EpiDISH. Shown are the boxplots of the average weight proportions of the major cell types epithelial cells (Epi), fibroblasts (Fib) and immune cells (IC) for EWAS (a) and OSCC samples (b). a EpiDISH estimations for the complete EWAS sample set (smokers and never smokers, $n=39)$ and for the subsets smokers $(n=18)$ and never smokers $(n=21)$. b EpiDISH estimations for the complete set of analysed OSCC samples (smokers and non-smokers, $n=16$ ) and for the subsets smokers ( $n=7$ ) and non-smokers $(n=9)$

Table 5 Differentially expressed transcripts in smokers compared to never smokers

\begin{tabular}{|c|c|c|c|c|}
\hline Ensembl gene & Gene symbol & $\log 2$ fold change & $p$ value & $q$ value \\
\hline ENSG00000138061 & $C Y P 1 B 1^{a}$ & 2.9 & $2.2 \times 10^{-14}$ & $4.9 \times 10^{-10}$ \\
\hline ENSG00000181019 & NQO1 & 1.2 & $1.2 \times 10^{-10}$ & $1.3 \times 10^{-6}$ \\
\hline ENSG00000176153 & GPX2 & 1.0 & $1.0 \times 10^{-9}$ & $7.7 \times 10^{-6}$ \\
\hline ENSG00000108602 & ALDH3A1 & 0.9 & $3.6 \times 10^{-9}$ & $2.0 \times 10^{-5}$ \\
\hline ENSG00000140465 & $C Y P 1 A 1^{\mathrm{a}}$ & 4.6 & $2.7 \times 10^{-8}$ & $1.2 \times 10^{-4}$ \\
\hline ENSG00000278270 & RPS9 & 2.3 & $3.1 \times 10^{-7}$ & 0.0012 \\
\hline ENSG00000164236 & ANKRD33B & -0.7 & $6.2 \times 10^{-7}$ & 0.0019 \\
\hline ENSG00000167165 & UGT1AG & 0.8 & $1.8 \times 10^{-6}$ & 0.0042 \\
\hline ENSG00000171403 & KRT9 & 1.3 & $1.9 \times 10^{-6}$ & 0.0042 \\
\hline ENSG00000180574 & EIF2S3B & -3.2 & $1.7 \times 10^{-6}$ & 0.0042 \\
\hline ENSG00000244122 & UGT1A7 & 1.6 & $3.0 \times 10^{-6}$ & 0.0060 \\
\hline ENSG00000197641 & SERPINB13 & 0.6 & $3.7 \times 10^{-6}$ & 0.0068 \\
\hline ENSG00000267023 & & 5.2 & $7.4 \times 10^{-6}$ & 0.0126 \\
\hline ENSG00000124588 & NQO2 & 0.4 & $1.1 \times 10^{-5}$ & 0.0179 \\
\hline ENSG00000117009 & KMO & 2.9 & $1.6 \times 10^{-5}$ & 0.0226 \\
\hline ENSG00000118322 & ATP10B & 0.5 & $1.6 \times 10^{-5}$ & 0.0226 \\
\hline ENSG00000284292 & & -2.8 & $2.6 \times 10^{-5}$ & 0.0335 \\
\hline ENSG00000135766 & EGLN1 & -0.3 & $3.0 \times 10^{-5}$ & 0.0368 \\
\hline ENSG00000119938 & PPPIR3C & -0.6 & $3.2 \times 10^{-5}$ & 0.0375 \\
\hline ENSG00000135299 & ANKRD6 & -0.4 & $4.5 \times 10^{-5}$ & 0.0497 \\
\hline
\end{tabular}

Differential expression analysis based on the RNA-Sequencing data. Log2 fold changes are given for the comparison smokers against never smokers ${ }^{a}$ Differentially methylated in our EWAS with $q<0.05$ 
Table 6 Results from the pathway analyses for differentially expressed genes

\begin{tabular}{|c|c|c|c|c|}
\hline Pathway & Identifier & Class & $p$ value & $q$ value \\
\hline \multicolumn{5}{|l|}{ Reactome database } \\
\hline The Citric Acid Cycle and Respiratory Electron Transport & R-HSA-1428517 & Metabolism (electron transport) & $1.4 \times 10^{-5}$ & 0.009 \\
\hline tRNA Aminoacetylation & R-HSA-379724 & Metabolism of proteins & $1.1 \times 10^{-4}$ & 0.019 \\
\hline Assembly of the pre-Replicative Complex & R-HSA-68867 & DNA replication & $1.3 \times 10^{-4}$ & 0.019 \\
\hline M-G1-Transition & NA & Cell cycle & $1.4 \times 10^{-4}$ & 0.019 \\
\hline Cytosolic tRNA Aminoacylation & R-HSA-379716 & Metabolism of proteins & $1.7 \times 10^{-4}$ & 0.019 \\
\hline HIV-Infection & R-HSA-162906 & Disease & $2.1 \times 10^{-4}$ & 0.019 \\
\hline Host Interactions of HIV Factors & R-HSA-162909 & Disease & $2.1 \times 10^{-4}$ & 0.019 \\
\hline Cell Cycle Checkpoints & R-HSA-69620 & Cell Cycle & $3.4 \times 10^{-4}$ & 0.021 \\
\hline Destabilisation of mRNA by AUF1 (hnRNP D0) & R-HSA-450580 & Metabolism of RNA & $3.5 \times 10^{-4}$ & 0.021 \\
\hline $\begin{array}{l}\text { Respiratory electron transport, ATP synthesis by } \\
\text { chemiosmotic coupling, and heat production by } \\
\text { uncoupling proteins }\end{array}$ & R-HSA-163200 & Metabolism (electron transport) & $3.7 \times 10^{-4}$ & 0.021 \\
\hline CDT1 association with the CDC6:ORC:origin complex & R-HSA-68827 & DNA replication & $3.7 \times 10^{-4}$ & 0.021 \\
\hline $\begin{array}{l}\text { Genes involved in APC/C:Cdc20 mediated degradation of } \\
\text { mitotic proteins }\end{array}$ & R-HSA-176409 & Cell cycle & $5.4 \times 10^{-4}$ & 0.026 \\
\hline Orc1 removal from chromatin & R-HSA-68949 & Cell cycle, DNA replication & $5.7 \times 10^{-4}$ & 0.026 \\
\hline Regulation of mitotic cell cycle & R-HSA-453276 & Cell cycle & $6.0 \times 10^{-4}$ & 0.026 \\
\hline Respiratory electron transport & R-HSA-611105 & Metabolism (electron transport) & $6.0 \times 10^{-4}$ & 0.026 \\
\hline G1/S Transition & R-HSA-69206 & Cell cycle & $7.3 \times 10^{-4}$ & 0.029 \\
\hline Regulation of ornithine decarboxylase (ODC) & R-HSA-350562 & Metabolism & $9.2 \times 10^{-4}$ & 0.033 \\
\hline CDK-mediated phosphorylation and removal Cdc6 & R-HSA-69017 & Cell cycle, DNA replication & $9.5 \times 10^{-4}$ & 0.033 \\
\hline Autodegradation of the E3 ubiquitin ligase COP1 & R-HSA-349425 & Cell cycle & $9.8 \times 10^{-4}$ & 0.033 \\
\hline $\begin{array}{l}\text { APC/C:Cdh1 mediated degradation of } \mathrm{Cdc} 20 \text { and other } \\
\text { APC/C:Cdh1 targeted proteins in late mitosis/early G1 }\end{array}$ & R-HSA-174178 & Cell cycle & $1.2 \times 10^{-3}$ & 0.038 \\
\hline Metabolism of RNA & R-HSA-8953854 & Metabolism of RNA & $1.3 \times 10^{-3}$ & 0.038 \\
\hline SCF-beta-TrCP mediated degradation of Emi1 & R-HSA-174113 & Cell cycle & $1.4 \times 10^{-3}$ & 0.039 \\
\hline Mitotic G1-G1/S phases & R-HSA-453279 & Cell cycle & $1.4 \times 10^{-3}$ & 0.040 \\
\hline Mitotic M-M/G1 phases & R-HSA-453274 & Cell cycle & $1.5 \times 10^{-3}$ & 0.040 \\
\hline \multicolumn{5}{|l|}{ KEGG database } \\
\hline Aminoacyl tRNA Biosynthesis & hsa00970 & Translation & $1.9 \times 10^{-4}$ & 0.034 \\
\hline Metabolism of Xenobiotics by Cytochrome P450 & rn00980 & Metabolism/xenobiotics biodegradation & $3.7 \times 10^{-4}$ & 0.034 \\
\hline
\end{tabular}

two significant CpGs mapping to $A H R R$ and $C Y P 1 B 1$, and another CpG $6 \mathrm{~kb}$ downstream of CYP1A2.

We consider the association of the non-coding RNA LINC00673 as another interesting finding. This lncRNA was significantly hypomethylated in smokers in our study at three individual CpGs. This gene was shown to be upregulated in tongue squamous cell carcinoma [38].

Differential methylation of $A H R R, C Y P 1 B 1$, and LINC00673 was shown before in buccal cells of smokers [22]. Increased expression of CYP1B1 by cigarette smoke was shown in vivo in buccal cells after smoke stimulation [39], in bronchial airway epithelial cells from smokers [40] and in vitro in A549 lung adenocarcinoma cells [23]. CYP1B1-AS1 was also shown to be upregulated in vitro by CSE exposure in A549 cells [23].
We could validate these findings in our differential expression analysis, showing a significant upregulation of $C Y P 1 B 1$, and additionally of $C Y P 1 A 1$ in the masticatory mucosa of smokers and a CSE-induced upregulation of $C Y P 1 B 1$ and $C Y P 1 B 1-A S$ in vitro. Likewise, pathway analysis of differentially expressed genes revealed a significant upregulation of genes involved in the Metabolism of Xenobiotics by Cytochrome P450-KEGG pathway. Smoking is a widespread risk factor for periodontitis, a common inflammatory disease of the supporting tissues of the oral cavity, which is characterised by severe inflammation of the gingiva and irreversible bone degradation leading to tooth loss. With the data provided by this study, we add evidence to the significance of smoking on differential DNA methylation of cytochrome-P450-mediated AhR- 
Table 7 Correlation of differential methylation with differences in transcript levels

\begin{tabular}{|c|c|c|c|c|c|c|c|c|c|c|}
\hline Gene & $p$ value (DE) & $q$ value (DE) & $\log 2 \mathrm{FC}$ & Best CpG & Chr & Position in bp (hg19a) & Add. CpGs & $p$ value $(\mathrm{DM})^{\mathrm{b}}$ & $q$ value (DM) & $\Delta \beta / 10 \mathrm{SPY}$ \\
\hline$\overline{C Y P 1 B 1}$ & $2.2 \times 10^{-14}$ & $1.3 \times 10^{-10}$ & 2.94 & cg01584760 & 2 & $38,296,474$ & 6 & $3.8 \times 10^{-10}$ & $3.1 \times 10^{-4}$ & -0.046 \\
\hline SLC7A5 & $1.1 \times 10^{-4}$ & $6.5 \times 10^{-2}$ & 0.62 & cg10099957 & 16 & $87,869,757$ & 2 & $9.6 \times 10^{-6}$ & $7.1 \times 10^{-2}$ & -0.060 \\
\hline$\angle A M A 3^{C}$ & 0.025 & 0.64 & -0.20 & cg16113793 & 18 & $21,451,607$ & 1 & $1.5 \times 10^{-6}$ & $4.0 \times 10^{-2}$ & -0.027 \\
\hline EDC3 & 0.031 & 0.65 & 0.39 & cg17181940 & 15 & $74,948,075$ & 1 & $5.6 \times 10^{-6}$ & $5.9 \times 10^{-2}$ & -0.054 \\
\hline
\end{tabular}

Genes nominally significant in the differential expression (DE) analysis that correspond to those CpGs that were associated with smoke pack years (SPY) with $q<$ 0.15 in the differential methylation (DM) analysis and that lie within genetic regions previously reported to be differentially methylated in buccals [22]. EDC3 was additionally reported to be differentially methylated in blood [32]

${ }^{\mathrm{a}} \mathrm{NCBI}$ build GRCh37

${ }^{\mathrm{b}} p$ values are from the analysis of covariance (ANCOVA) with adjustment by age, sex and batch. DE differential expression, Log2FC log2fold change, Chr chromosome, $b p$ basepairs, Add. CpGs additional CpGs significant with $q<0.15$ at this locus, DM differential methylation, SPY smoke pack years

'Effect sizes for DE and DM are contradictory

pathway, a pathway that is involved in smoke-mediated bone degradation [37].

Apart from the upregulation of the Cytochrome P450related pathway, Reactome pathway analysis of differentially expressed genes found 58\% (14 out of 24) altered pathways relating to the classes DNA replication or cell cycle function, suggesting a connection of smoking and tumorigenesis.

Our data did not suggest a global correlation of the results from the differential methylation analysis with the differential expression analysis. However, we note that the majority of effect sizes in smoking EWAS are not large, with $12.5 \%$ hypomethylation in smokers for $A H R R$ in our study. Therefore, the identification of subtle changes in expression caused by the methylation differences detected in EWAS requires very large sample sizes, even if assumed that the relationship between methylation and expression is linear. When accounting for other regulatory mechanisms that disturb this hypothesised linearity, the detection of a direct relationship between changes in methylation patterns and changes in expression patterns becomes even more

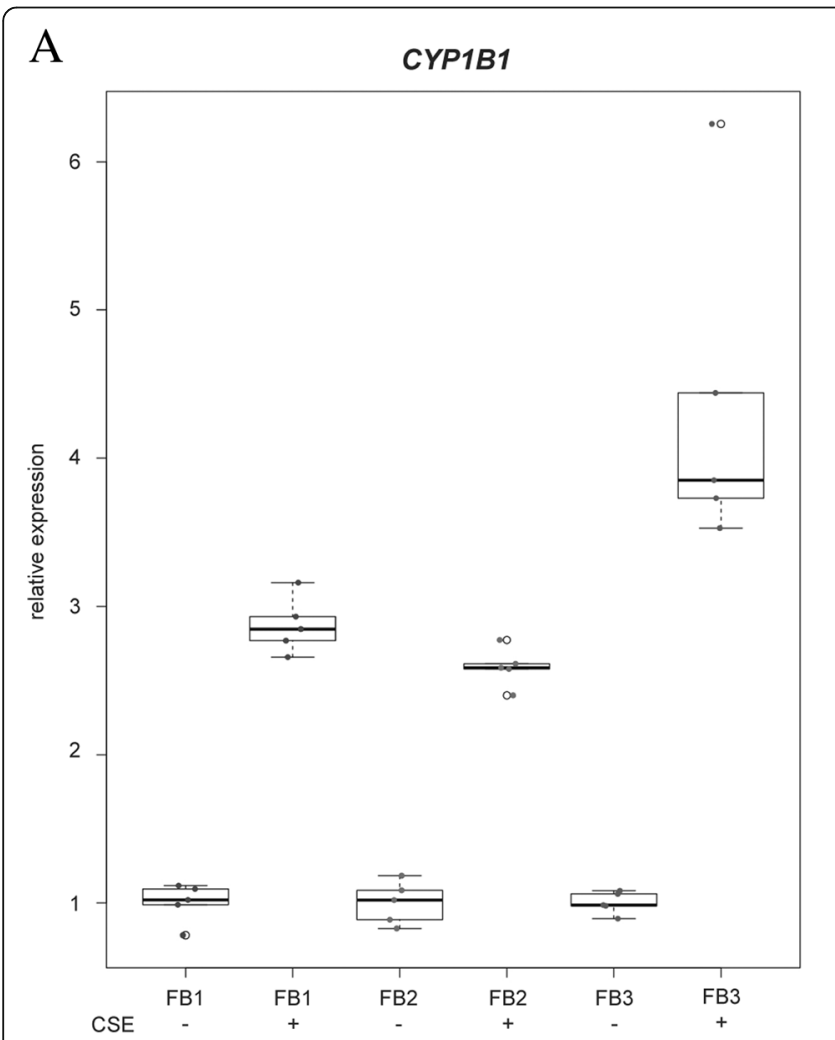

B

CYP1B1-AS

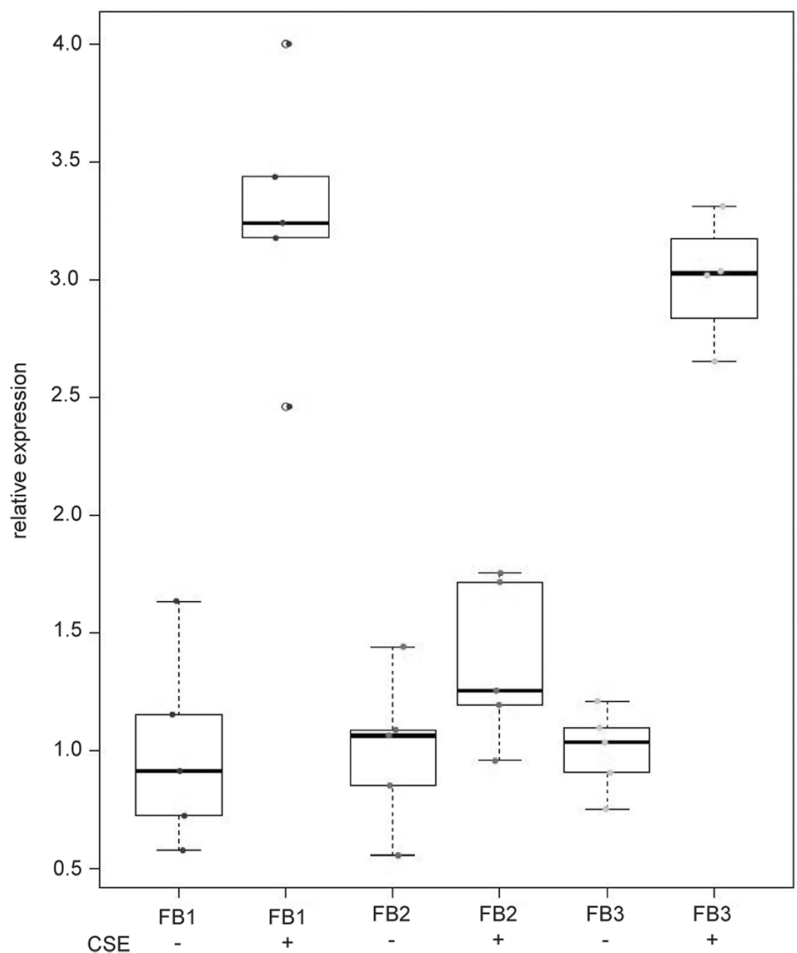

Fig. 6 Gene expression of CYP1B1 and CYP1B1-AS is significantly increased by cigarette smoke extract in vitro. Primary gingival fibroblast cell lines were cultured from three independent ex vivo biopsies (FB1-3) and treated for $6 \mathrm{~h}$ with cigarette smoke extract (CSE), labelled as " + ". Control cell lines without CSE treatment are labelled as " $-"$ ". qRT-PCR data were obtained from five treatments with CSE. Significant upregulation upon CSE treatment were observed for CYP1B1 ( $\left.p_{\text {adj }}=0.019\right)$ and CYP1B1-AS ( $\left.p_{\text {adj }}=0.023\right)$. $P$ values are Bonferroni-adjusted for two independent tests 
Table 8 Associations of smoking and cancer with differential methylation of AHRR and CYP1B1

\begin{tabular}{|c|c|c|c|c|c|c|c|c|c|c|c|c|}
\hline & \multirow[t]{2}{*}{ CpG } & \multirow[t]{2}{*}{ Chr } & \multirow[t]{2}{*}{$\begin{array}{l}\text { Position in } \\
\text { bp }\left(\text { hg } 19^{a}\right)\end{array}$} & \multicolumn{3}{|c|}{$\begin{array}{l}\text { Association with SPY (EWAS } \\
\text { samples) }\end{array}$} & \multicolumn{3}{|c|}{$\begin{array}{l}\text { Association with smoking status } \\
\text { (EWAS samples) }\end{array}$} & \multicolumn{3}{|c|}{ Association with OSCC } \\
\hline & & & & $\Delta \beta / 10$ SPY & $p_{\text {nom }}$ & 9 & $\Delta \beta$ & $p_{\text {nom }}$ & 9 & $\Delta \beta$ & $p_{\text {nom }}$ & $p_{\text {adj }}$ \\
\hline \multirow[t]{5}{*}{ CYP1B1 } & cg01584760 & 2 & $38,296,474$ & -0.046 & $3.8 \times 10^{-10}$ & $3.1 \times 10^{-4}$ & -0.045 & $1.0 \times 10^{-4}$ & 0.853 & \multicolumn{3}{|c|}{ Not analysed } \\
\hline & cg02162897 & 2 & $38,300,537$ & -0.11 & $1.2 \times 10^{-7}$ & 0.014 & -0.183 & $5.5 \times 10^{-8}$ & 0.022 & 0.197 & $4.7 \times 10^{-6}$ & $6.1 \times 10^{-4}$ \\
\hline & cg20408276 & 2 & $38,300,586$ & -0.108 & $1.1 \times 10^{-7}$ & 0.014 & -0.176 & $3.6 \times 10^{-7}$ & 0.04 & \multicolumn{3}{|c|}{ Not analysed } \\
\hline & cg00565882 & 2 & $38,300,707$ & -0.041 & $1.7 \times 10^{-6}$ & 0.042 & -0.044 & 0.005 & 1 & 0.16 & $8.6 \times 10^{-7}$ & $1.1 \times 10^{-4}$ \\
\hline & cg01410359 & 2 & $38,302,230$ & -0.037 & $3.4 \times 10^{-6}$ & 0.047 & -0.032 & 0.023 & 1 & 0.177 & $8.8 \times 10^{-7}$ & $1.1 \times 10^{-4}$ \\
\hline \multirow[t]{4}{*}{$A H R R$} & cg04551776 & 5 & 393,366 & -0.007 & $4.3 \times 10^{-1}$ & 0.97 & -0.011 & 0.43 & 1 & 0.149 & $2.7 \times 10^{-5}$ & $3.5 \times 10^{-3}$ \\
\hline & cg06036945 & 5 & 394,869 & -0.067 & $1.0 \times 10^{-4}$ & 0.18 & -0.125 & $3.7 \times 10^{-7}$ & 0.04 & \multicolumn{3}{|c|}{ Not analysed } \\
\hline & cg04066994 & 5 & 394,891 & -0.041 & $8.2 \times 10^{-6}$ & 0.067 & -0.083 & $5.9 \times 10^{-10}$ & $4.7 \times 10^{-4}$ & \multicolumn{3}{|c|}{ Not analysed } \\
\hline & cg25648203 & 5 & 395,444 & -0.007 & 0.42 & 0.99 & -0.012 & 0.37 & 1 & -0.081 & $9.7 \times 10^{-3}$ & 1 \\
\hline
\end{tabular}

Shown are the results for all CpGs within CYP1B1 and AHRR that were significantly associated with SPY levels or smoking status in our EWAS. We compared AHRR and CYP1B1 methylation levels in 16 oral squamous cell carcinoma (OSCC) samples from TCGA to our healthy never smoker samples. Differential methylation analysis was performed using an ANCOVA adjusting for sex and unknown variation. The CpGs cg01584760, cg20408276, cg06036945 and cg04066994 were not covered by the 450k analysis of the OSCC samples. For AHRR, we added the results for cg04551776 and cg25648203, which flanked our EWAS-CpGs with a distance of $1503 \mathrm{bp}$ and $553 \mathrm{bp}$, respectively, albeit these CpGs were not associated in our EWAS

${ }^{a}$ NCBI build GRCh37

${ }^{\mathrm{b}}$ Top significant $\mathrm{CpG}$ in the differential methylation analysis with SPY

${ }^{{ }^{C} T o p}$ significant $\mathrm{CpG}$ in the differential methylation analysis with smoking status. Chr chromosome, bp basepairs, SPY smoke pack years, $P_{\text {adj }}$ Bonferroni-adjusted for 130 tests

difficult. Accordingly, EWAS that identified a large correlation of significant methylation with transcription differences are rare, and this despite the fact that hypomethylation is functionally associated with increased gene expression. However, we are aware of a large study that addressed the effects of smoking on methylation and transcription in 730 blood samples, which identified 254 CpGs showing significant correlation of differential methylation with expression values of nearby transcripts [13]. We presume that our non-finding of a global correlation is probably due to the lack of statistical power of the comparably small sample size. When filtering our expression data for genes with multiple associated CpGs and taking into account knowledge on previously published differentially methylated CpGs, in addition to CYP1B1, we identified two further genes, SLC7A5 and EDC3, that showed consistent effect sizes for differential methylation and differential expression, and which were supported by smoking EWAS on buccal cells or blood. Variants within $E D C 3$ have been reported in a large meta-analysis on caffeine intake, together with $A H R, C Y P 1 A 1$ and $C Y P 1 A 2$ [41]. EDC3 is located $25 \mathrm{~kb}$ upstream of CYP1A1 and $55 \mathrm{~kb}$ upstream of $C Y P 1 A 2$, which also harboured an associated CpG $6 \mathrm{~kb}$ upstream in our EWAS. Taken together, our results point towards an important role of differential methylation of several genes of the CYP-gene cluster on chromosome 15 to regulate the AhR-pathway in the masticatory mucosa of smokers.

In the comparison of CYP1B1 methylation patterns of our never smokers data with the OSCC samples, all five significant CpGs were hypomethylated in our EWAS but in the OSCC data, CYP1B1 showed global hypermethylation
(16 out of 17 significant CpGs). For AHRR, findings are less clear because none of our CPG was analysed on the 450k chip used for the OSCC samples. Likewise, in 51 OSCC samples from various extraction sites (e.g. buccal mucosa, floor of mouth, lip, palate), significant downregulation of CYP1B1 transcript levels was reported [42]. This contradicts the observation that CYP1B1 was overexpressed in many cancers [43]. As suggested earlier [22, 23], our observations may indicate that demethylation and upregulation of CYP1B1 in the oral mucosal tissues of smokers is not causal for the development of cancer, but in contrast, part of the normal cellular detoxification. In oral cancer, these pathways might be misregulated, which in turn would lead to impaired regulation of xenobiotic metabolism. However, these results contradict findings from other cancer types, where CYP1B1 is overexpressed and serves as a tumour marker [44]. It needs to be emphasised that a major limitation of the OSCC dataset is that extraction sites were not specified. Cell compositions vary among different regions of the oral cavity [45], which is also suggested by the results of our cell type deconvolution analysis, and which could account for a stratification of results. As OSCC and other oral tissue samples from TCGA most likely have a heterogeneous cellular background originating from diverse tissue extraction sites, they might not be a perfect data source for a straightforward identification of differential methylation patterns. To investigate cell type-specific and disease-relevant methylation patterns in specific parts of the oral mucosa, further cell type deconvolution studies from different extraction sites and cell types of the oral mucosa can improve future analyses. The main limitation of our EWAS was the small sample size. Yet, our samples 
allowed the detection of the most significant tobacco smoke-related differentially methylated loci, which indicates the power of this homogeneously collected sample set.

\section{Conclusion}

The current study adds evidence to previous EWAS that reported significant hypomethylation of $A H R R$ and $C Y P 1 B 1$ in buccal and airway epithelium of smokers. We could show increased expression of CYP1B1 and $C Y P 1 B 1-A S$ in the masticatory mucosa of smokers as compared to never smokers and validated upregulation of these genes by cigarette smoke in vivo and in vitro. We conclude that hypomethylation of $A H R R$ and $C Y P 1 B 1$ is an important regulatory mechanism of xenobiotic metabolism of the masticatory mucosa in response to tobacco smoke exposure.

\section{Methods}

\section{Study sample}

A total of 39 healthy smokers and never smokers, i.e. free of systemic disease, and all of European descent, were enrolled in this study, which was conducted in accordance with the principles of Good Clinical Practice and approved by the Independent Ethics Committee of each participating University Hospital. Written informed consent was obtained from all subjects according to the Declaration of Helsinki. All study participants completed a detailed questionnaire to provide general personal information (e.g. sex, age, geographical and family descent), information on the general and oral health and smoking habits (current, former, never smoker and amount of smoking in cigarette packs per day and duration of smoking in years). We calculated smoking pack year (SPY) values by multiplying the number of smoked cigarette packs per day by the number of years smoked. Fifty percent of the smokers had $>10$ SPY, and 50\% had between two and ten SPY. The mean age in smokers was 38 years and 35 years in never smokers. All individuals underwent periodontal examination prior to tissue extraction to exclude the presence of PD. Only individuals with a periodontal screening index $(\mathrm{PSI})<2$ were included, indicating the absence of severe oral inflammation.

\section{Collection of ex-vivo tissue samples from the masticatory oral mucosa}

Solid tissue samples from the oral masticatory mucosa were collected at a defined site from the hard palate adjacent to the 4th and 5th tooth by the use of a tissue puncher with $3 \mathrm{~mm}$ diameter and a depth of approximately $1 \mathrm{~mm}$ (for an illustration of the area of tissue sampling, see Additional file 7: Figure S1). We consider this site also representative for the gingival mucosa (i.e. often inflamed in periodontitis) because the gingival masticatory mucosa and the masticatory mucosa of the hard palate share the same histological cellular structures (Stratum basale, Str. spinosum, Str. granulosum, Str. corneum) and therefore the same cell types (Additional file 7: Figure S2). Likewise, indicating similar states of cellular differentiation, tissue collection at the specified sampling area is a common approach during periodontal surgery when free gingival grafts are excised and transplanted to other mucosal areas in the oral cavity. To stabilise DNA and RNA, the biopsies were stored in the AllProtect reagent (Qiagen, Hilden, Germany) immediately after punching, stored at $4{ }^{\circ} \mathrm{C}$ for $24 \mathrm{~h}$, and subsequently transferred to $-20{ }^{\circ} \mathrm{C}$.

\section{DNA and RNA extraction}

The conserved tissue samples were manually broken up into small pieces with a scalpel and subsequently homogenised using the automated mixer mill MM 400 (Retsch, Haan, Germany) using frozen beads (3 mm, Retsch) for $90 \mathrm{~s}$ at $30 \mathrm{~Hz}$. Subsequently, DNA and RNA was extracted simultaneously using the AllPrep DNA/RNA/miRNA Universal Kit (Qiagen, Hilden, Germany), ensuring the same cellular background of DNA and RNA for subsequent comparison of DNA-methylation and RNAexpression. Integrity of DNA and RNA was subsequently verified with a $2 \%$ agarose gel electrophoresis. Concentrations were measured using the Multiskan GO Microplate Spectrophotometer (Fisher Scientific, Hampton, USA). DNA and RNA samples were stored at $-80^{\circ} \mathrm{C}$.

\section{Bisulfite conversion and hybridisation to the Infinium MethylationEPIC BeadChips}

Five hundred nanograms DNA per sample was bisulfite converted with the EZ-96 DNA Methylation Kit (Zymo Research, Irvine, USA) and hybridised to the Infinium DNA MethylationEPIC BeadChip (Illumina, San Diego, USA) on an iScan Microarray Scanner (Illumina) at the Institute for Clinical Molecular Biology, ChristianAlbrechts-University Kiel, Germany.

\section{Differential DNA methylation analysis}

For analysis and quality control, the $\mathrm{R}$ environment (Version 3.5.1) and the $\mathrm{R}$ package minfi (1.26.2) [46] were used, including the $\mathrm{R}$ package limma (Version 3.36.5) for the differential methylation analysis [47]. The Red/Green channel of the array were converted into one methylation signal without any normalisation. Using the function plotQC, we estimated sample-specific quality control $(\mathrm{QC})$ and did not remove any sample. The QC criteria for probes were filtering of probes with median detection $p$ values $>0.05$, probes that lay within 5 basepairs (bp) of a single nucleotide polymorphism (SNP) 
with $>5 \%$ minor allele frequency, probes on the sex chromosomes and cross-reactive probes using the $\mathrm{R}$ package maxprobes (https://rdrr.io/github/markgene/ maxprobes) according to Pidsley et al. [48] and McCartney et al. [49]. In total, 802,254 probes complied with the QC criteria and were included in the analysis. Methylation status was estimated according to the fluorescent intensity ratio, as any value between $\beta=0$ (unmethylated) and 1 (completely methylated), and $\log 2-$ transformed into $M$ values, which are considered a more statistically valid estimator [50]. Corresponding effect sizes are, for a better biological understanding, given as $\Delta \beta$, unless otherwise stated. After quality control, we performed a functional normalisation using the preprocessFunnorm function in minfi [51]. To identify CpGs correlating significantly with SPY, an analysis of covariance (ANCOVA) was performed for SPY as a continuous variable with adjustment by age as a continuous variable and sex as a categorical variable. We used SPY in this model to account for the cumulative risk exposure conferred by long-term smoking as suggested by Teschendorff et al. 2015 [22]. We additionally investigated differential methylation correlating with smoking status by performing an ANCOVA with the covariates age and sex. In both analyses, we additionally adjusted for technical variation, i.e. slide and position on slide, by using Combat and the R package BatchQc [52]. Correction for multiple testing was performed using the method by Benjamini \& Hochberg [53]. CpGs were annotated to genes according to GRCh37/hg19 as provided in the MethylationEPIC BeadChip manifest.

\section{Cell type deconvolution of EWAS samples}

To identify the presence of non-epithelial cells in our samples of oral mucosa, we used the EpiDISH algorithm (Version 3.9) [54], applying the centEpiFibIC.m.rda reference dataset on our beta-values, which includes centroids for epithelial cells, fibroblasts and immune cells.

\section{RNA-sequencing}

To validate the effects of the observed methylation differences between smokers and never smokers on gene expression in the oral mucosa, we performed RNA-sequencing (RNA-Seq) of the same samples that were used in the EWAS. This ensured a homogeneous genetic and cellular background of the methylation and expression analysis. The total mRNA of 38 biopsy samples that passed QC for RNA-Seq (> 600 ng of total RNA, RIN > 7) was sequenced with a coverage of 16 million 50 bp single-end reads per sample. One RNA-sample was discarded due to low amounts of RNA. In brief, the library preparation was performed with $600 \mathrm{ng}$ RNA using the TruSeq
Stranded mRNA Kit (Illumina). RNA-Seq was performed on a HiSeq3000 (Illumina) at the Institute for Clinical Molecular Biology of the Christian-AlbrechtsUniversity Kiel, Germany.

\section{Differential expression analysis}

RNA-seq samples were mapped to the transcriptome (Ensembl 96, GRCh38) using the transcript abundance quantification method Salmon [55]. Salmon estimates abundances without aligning reads. The transcript level estimates were then summarised for the gene level and imported into $\mathrm{R}$ using the package tximport [56]. Subsequently, the imported data was analysed for differential expression between smokers and never smokers using DESeq2 [57]. Genes with $\leq 2$ counts in all samples combined were not considered in the analyses. To identify genes that show substantial evidence for a correlation of differential expression with differential methylation in smokers, we applied the following steps: (1) filtering of all differentially expressed transcripts with unadjusted $p<0.05$; (2) filtering of transcripts with more than one CpG associated with $\mathrm{q}<0.15$ with SPY; (3) filtering of transcripts that were previously reported in a large EWAS on buccal swabs [22] or in a systematic review on EWAS on blood [32].

\section{Gene set enrichment analysis of differentially expressed genes}

Gene sets of KEGG $(n=186)$ and Reactome $(n=674)$ were gathered from the Broad Institute Molecular Signatures Database (MSigDB [58, 59]). Together with the $\log 2$ FoldChanges of the differential expression analysis, we performed an enrichment analysis using the $\mathrm{R}$ package gage [60]. Gene sets with $q$ value $<0.05$ were considered as being significantly enriched.

\section{Preparation of cigarette smoke extract and RNA extraction of cultured cells}

Cigarette smoke extraction system and cigarette smoke extract (CSE) were prepared as described in [61]. CSE was prepared from Roth-Händle no filters cigarettes (Reemtsma, Hamburg, Germany). Smoke of three cigarettes was filtered using a $0.2-\mu \mathrm{M}$ sterile filter and extracted into $10 \mathrm{~mL}$ cell culture medium. The CSEcontaining medium was directly added to the cells (3 mL per well).

\section{CSE stimulation of primary gingival fibroblasts}

For CSE stimulation, solid ex vivo biopsies of the masticatory oral mucosa of the hard palate were taken from three additional participants with $3 \mathrm{~mm}$ tissue punchers. Biopsies were dissected enzymatically to separate the epithelial cells from the fibroblasts by overnight incubation in $5 \mathrm{mg} / \mathrm{mL}$ dispase II (Sigma Aldrich) diluted in cell 
growth medium (DMEM, $1 \%$ Pen/Strep) at $4{ }^{\circ} \mathrm{C}$. The primary gingival fibroblast cells (pGFs) were subsequently cultured in cell growth medium (DMEM, 1\% Amphotericin B, $1 \%$ Pen/Strep, $1 \%$ non-essential amino acids). Prior to CSE stimulation, the pGFs (passage 3-4) were seeded in 6-well tissue culture plates (TPP Techno Plastic Products, Trasadingen, Switzerland) $24 \mathrm{~h}$ before stimulation $\left(2.2 \times 10^{5}\right.$ cells per well). The CSE was freshly prepared at the day of stimulation. CSE induction was performed for $6 \mathrm{~h}$ in three biological replicates (each with five technical replicates) of pGFs, with aliquots of the same CSE. Three millilitre medium without CSE was added to the control cell plates (five technical replicates for each of the three donors). After $6 \mathrm{~h}$ of CSE incubation, the cells were washed twice with PBS. Cell disruption and total RNA extraction was carried out using the RNeasy Mini Kit (Qiagen) according to the manufacturer's instructions.

\section{CDNA synthesis and qRT-PCR}

To test differential expression in primary gingival fibroblasts upon CSE-stimulation, we performed quantitative reverse transcription polymerase chain reaction (qRTPCR) for CYP1B1 and CYP1B1-AS. qRT-PCR was performed with an amount of $500 \mathrm{ng}$ of total RNA of CSE-stimulated cells using the High-Capacity cDNA Reverse Transcription Kit and oligo-(dT)-primers (Thermo Fisher Scientific, Waltham, USA) in accordance with the manufacturer's guidelines, using oligo-(dT)- and dNTPprimers instead of random primers. Control PCR reactions contained water instead of cDNA. qRT-PCR experiments were performed using the CFX Connect System (Bio-Rad, Hercules, USA) in combination with SYBR Select Master Mix (Thermo Fisher Scientific) according to the manufacturer's instructions. The gene expression levels were normalised to the mRNA expression of $G A P D H$, and relative expression was calculated using the mathematical model delta-delta ct (GraphPad Prism Software/R). Primers were manufactured (metabion $\mathrm{GmbH}$, Planegg/Steinkirchen, Germany) with the following sequences: CYP1B1 (forward: GAC GAC CCC GAG TTC CGT GA; reverse: AGC CAG GGC ATC ACG TCC AC) and CYP1B1-AS (forward: AGC CCT GAA AGA TGA ACA GTG GT; reverse: GGC ATG CCC ATT TCT TCC ACA). Because the lncRNA CYP1B1-AS1 was not covered by mRNA-Sequencing, we analysed expression in our exvivo biopsies from the EWAS and 17 additional biopsies that were collected as described above with qRT-PCR. In total, CYP1B1-AS-expression of 32 smokers and 37 never smokers was analysed.

\section{Cell type deconvolution and differential methylation analysis of OSCC samples}

To identify a putative role of genes that showed differential methylation in oral mucosa of smokers in cancer- development, we analysed data from The Cancer Genome Atlas (TCGA) [62]. We downloaded Illumina 450k methylation bead chip data from 16 samples of oral squamous cell carcinoma (OSCC) of keratinized oral mucosa (sites specified as "palate" or "gums"; i.e., Hard Palate, Upper Gum, Lower Gum and Gum NOS in the TCGA). To account for cell type-specific methylation, we performed the cell type deconvolution analyses on the 450k OSCC data as described above. We then performed an ANCOVA for all $130 \mathrm{CpGs}$ that were annotated on the Illumina $450 \mathrm{k}$ array to the genetic regions of CYP1B1 and AHRR with sex as a covariate and adjusting for technical variation using the $\mathrm{R}$ package sva [63] to compare OSCC samples to the healthy never smokers from the EWAS.

\section{Additional files}

Additional file 1: CpGs from the literature associated with SPY in our data. Results are shown for the ANCOVA with SPY for all CpGs with $q<$ 0.15 in our EWAS that map to genes harbouring a significant CpG in an EWAS on buccal swabs [22] or were reported as replicated in at least two independent EWAS on blood [32]. (XLSX 47 kb)

Additional file 2: Results of the EpiDISH cell type deconvolution. Estimates for the different cell type fractions are given each for the EWAS samples and the OSCC samples, each also additionally divided into the subsets smokers and non-smokers. (XLSX $17 \mathrm{~kb}$ )

Additional file 3: Median TPM values from RNA-Sequencing. Transcripts were ranked according to their median TPM value in the EWAS samples, with the highest expressed genes on top. (XLSX $1299 \mathrm{~kb}$ )

Additional file 4: Results from the KEGG and Reactome Pathway Analyses of Differentially Expressed Genes. (XLSX 76 kb)

Additional file 5: Correlation of associated CpGs with association of transcripts. Shown are all CpGs with $q<0.15$ that map to genes that were differentially expressed in smokers with a nominal $p$ value of $<0.05$. (XLSX $14 \mathrm{~kb}$ )

Additional file 6: Results from the ANCOVA of OSCC samples in AHRR and CYP1B1. Listed are all CpGs within the genetic regions of AHRR and CYP1B1 $(n=202)$ to indicate absence of CpGs in the $450 \mathrm{k}$ analysis of OSCC. CpGs passing the adjusted significance threshold are marked in red. (XLSX $8961 \mathrm{~kb})$

Additional file 7: Figure S1. Area of tissue sampling. The tissue samples were collected from the hard palate directly adjacent to the 4th and 5 th tooth by the use of a tissue puncher ( $3 \mathrm{~mm}$ diameter). Figure S2. Histological features of the masticatory mucosa of the gingiva and the hard palate. The mucosa of both sites is characterised by the four layered orthokeratinised stratified squamous epithelium, overlaying the lamina propria. The lamina propria contains closely packed bundles of collagen fibres enabling the mucosa to resist heavy loading. The cells in the upper keratin layer have lost their nuclei. Because the function and appearance of the cells and cell layers from both extraction sites are similar, it is the broad agreement of the periodontologists participating in this study that both sites are comparable and are likely to share similar methylation patterns under normal conditions. (DOCX 1137 kb)

\section{Acknowledgements}

The results published for the OSCC analysis are in parts based upon data generated by the TCGA Research Network: https://www.cancer.gov/tcga. 
Bildung und Forschung (01DL15002), and a grant of the DG PARO/CP GABAForschungsförderung.

\section{Availability of data and materials}

The generated datasets from RNA-Sequencing experiment will be made publicly available on and the gene expression omnibus (GEO) database (https:// www.ncbi.nlm.nih.gov/geo/) upon acceptation for publication. DNA methylation data from the EWAS experiment will be available from the corresponding author on reasonable request.

\section{Authors' contributions}

GR and AS conceived and designed the study, interpreted the data and obtained third-party funding. HD, CB, YJS and OM recruited the study participants and collected the biopsies. GR processed the biopsies in the laboratory. AF provided the infrastructure and supervised the laboratory work for the methylation arrays. $\mathrm{RH}$ provided the infrastructure and supervised the laboratory work for the RNA-Sequencing. GR analysed the data. JK performed the statistical analyses of the EWAS. MM analysed the RNA-Seq data and performed the differential expression analyses. RW carried out the in vitro studies. JK, RW and GR created the figures for the manuscript. GR and AS wrote the manuscript and supervised the study. All authors helped draft the manuscript, participated in the manuscript editing and read/approved the final manuscript.

\section{Ethics approval and consent to participate}

This study was conducted in accordance with the principles of Good Clinical Practice and approved by the Independent Ethics Committee of the participating University Hospitals of Berlin (Germany), Würzburg (Germany), Vienna (Austria) and Coimbra (Portugal). Written informed consent was obtained from all subjects according to the Declaration of Helsinki.

\section{Consent for publication}

Not applicable.

\section{Competing interests}

The authors declare that they have no competing interests.

\section{Publisher's Note}

Springer Nature remains neutral with regard to jurisdictional claims in published maps and institutional affiliations.

\section{Author details \\ 'Department of Periodontology and Synoptic Dentistry, Institute for Dental and Craniofacial Sciences, Charité - Universitätsmedizin Berlin, Freie Universität Berlin, Humboldt-Universität zu Berlin, and Berlin Institute of Health, Aßmannshauser Str. 4-6, 14197 Berlin, Germany. ${ }^{2}$ Institute for Biometry and Clinical Epidemiology, Charité - Universitätsmedizin Berlin, Freie Universität Berlin, Humboldt-Universität zu Berlin, and Berlin Institute of Health, Charitéplatz 1, 10117 Berlin, Germany. ${ }^{3}$ Medical Systems Biology Group, Institute of Experimental Dermatology, Institute for Cardiogenetics, University of Lübeck, 23562 Lübeck, Germany. Institute of Clinical Molecular Biology, Christian-Albrechts-University, Rosalind-Franklin-Straße 12, 24105 Kiel, Germany. ${ }^{5}$ Institute of Periodontology, Dentistry Department, Faculty of Medicine, University of Coimbra, Av. Bissaya Barreto, Bloco de Celas, 3000-075 Coimbra, Portugal. ${ }^{6}$ Department of Periodontology, Clinic of Preventive Dentistry and Periodontology, University Medical Center of the Julius-Maximilians-University, Pleicherwall, 97070 Würzburg, Germany. ${ }^{7}$ Department of Conservative Dentistry and Periodontology, Medical University Vienna, School of Dentistry, Sensengasse 2a, 1090 Vienna, Austria.}

Received: 23 January 2019 Accepted: 18 June 2019

Published online: 22 July 2019

\section{References}

1. Thun MJ, Carter BD, Feskanich D, Freedman ND, Prentice R, Lopez AD, et al. 50-year trends in smoking-related mortality in the United States. N Engl J Med. 2013:368(4):351-64.

2. Ferreira Antunes $J$, Toporcov $T N$, Biazevic MG, Boing AF, Scully $C$, Petti $S$. Joint and independent effects of alcohol drinking and tobacco smoking on oral cancer: a large case-control study. PLoS One. 2013;8(7):e68132.
3. Tonetti MS, Claffey N, European Workshop in Periodontology group C Advances in the progression of periodontitis and proposal of definitions of a periodontitis case and disease progression for use in risk factor research. Group C consensus report of the 5th European Workshop in Periodontology. J Clin Periodontol. 2005;32(Suppl 6):210-3.

4. Portela A, Esteller M. Epigenetic modifications and human disease. Nat Biotechnol. 2010;28(10):1057-68.

5. Feinberg AP. Phenotypic plasticity and the epigenetics of human disease. Nature. 2007;447(7143):433-40.

6. Fraga MF, Ballestar E, Paz MF, Ropero S, Setien F, Ballestar ML, et al. Epigenetic differences arise during the lifetime of monozygotic twins. Proc Natl Acad Sci U S A. 2005;102(30):10604-9.

7. Flanagan JM. Epigenome-wide association studies (EWAS): past, present, and future. Methods Mol Biol. 2015;1238:51-63.

8. Ambatipudi S, Cuenin C, Hernandez-Vargas H, Ghantous A, Le Calvez-Kelm F, Kaaks R, et al. Tobacco smoking-associated genome-wide DNA methylation changes in the EPIC study. Epigenomics. 2016;8(5):599-618.

9. Breitling LP, Yang R, Korn B, Burwinkel B, Brenner H. Tobacco-smokingrelated differential DNA methylation: $27 \mathrm{~K}$ discovery and replication. Am J Hum Genet. 2011;88(4):450-7.

10. de Vries M, van der Plaat DA, Nedeljkovic I, Verkaik-Schakel RN, Kooistra W, Amin $\mathrm{N}$, et al. From blood to lung tissue: effect of cigarette smoke on DNA methylation and lung function. Respir Res. 2018;19(1):212.

11. Guida F, Sandanger TM, Castagne R, Campanella G, Polidoro S, Palli D, et al. Dynamics of smoking-induced genome-wide methylation changes with time since smoking cessation. Hum Mol Genet. 2015;24(8):2349-59.

12. Jessen WJ, Borgerding MF, Prasad GL. Global methylation profiles in buccal cells of long-term smokers and moist snuff consumers. Biomarkers. 2018; 23(7):625-39.

13. Joubert BR, Haberg SE, Nilsen RM, Wang X, Vollset SE, Murphy SK, et al. 450K epigenome-wide scan identifies differential DNA methylation in newborns related to maternal smoking during pregnancy. Environ Health Perspect. 2012;120(10):1425-31.

14. Philibert RA, Beach SR, Brody GH. Demethylation of the aryl hydrocarbon receptor repressor as a biomarker for nascent smokers. Epigenetics. 2012; 7(11):1331-8.

15. Rzehak P, Saffery R, Reischl E, Covic M, Wahl S, Grote V, et al. Maternal smoking during pregnancy and DNA-methylation in children at age 5.5 years: epigenome-wide-analysis in the European childhood obesity project (CHOP)-study. PLoS One. 2016;11(5):e0155554.

16. Sayols-Baixeras S, Lluis-Ganella C, Subirana I, Salas LA, Vilahur N, Corella D, et al. Identification of a new locus and validation of previously reported loci showing differential methylation associated with smoking. The REGICOR study. Epigenetics. 2015;10(12):1156-65.

17. Shenker NS, Polidoro S, van Veldhoven K, Sacerdote C, Ricceri F, Birrell MA, et al. Epigenome-wide association study in the European Prospective Investigation into Cancer and Nutrition (EPIC-Turin) identifies novel genetic loci associated with smoking. Hum Mol Genet. 2013;22(5):843-51.

18. Sun YV, Smith AK, Conneely KN, Chang Q, Li W, Lazarus A, et al. Epigenomic association analysis identifies smoking-related DNA methylation sites in African Americans. Hum Genet. 2013;132(9):1027-37.

19. Tsai PC, Glastonbury CA, Eliot MN, Bollepalli S, Yet I, Castillo-Fernandez JE, et al. Smoking induces coordinated DNA methylation and gene expression changes in adipose tissue with consequences for metabolic health. Clin Epigenetics. 2018;10(1):126.

20. Tsaprouni LG, Yang TP, Bell J, Dick KJ, Kanoni S, Nisbet J, et al. Cigarette smoking reduces DNA methylation levels at multiple genomic loci but the effect is partially reversible upon cessation. Epigenetics. 2014;9(10):1382-96.

21. Wan ES, Qiu W, Carey VJ, Morrow J, Bacherman H, Foreman MG, et al. Smoking-associated site-specific differential methylation in buccal mucosa in the COPDGene study. Am J Respir Cell Mol Biol. 2015;53(2):246-54.

22. Teschendorff $A E$, Yang Z, Wong A, Pipinikas $C P$, Jiao $Y$, Jones $A$, et al. Correlation of smoking-associated DNA methylation changes in buccal cells with DNA methylation changes in epithelial cancer. JAMA Oncol. 2015;1(4): 476-85.

23. Stueve TR, Li WQ, Shi J, Marconett CN, Zhang T, Yang C, et al. Epigenomewide analysis of DNA methylation in lung tissue shows concordance with blood studies and identifies tobacco smoke-inducible enhancers. Hum Mol Genet. 2017;26(15):3014-27.

24. Monick MM, Beach SR, Plume J, Sears R, Gerrard M, Brody GH, et al. Coordinated changes in AHRR methylation in lymphoblasts and pulmonary 
macrophages from smokers. Am J Med Genet B Neuropsychiatr Genet. 2012:159B(2):141-51.

25. Zeilinger S, Kuhnel B, Klopp N, Baurecht H, Kleinschmidt A, Gieger C, et al. Tobacco smoking leads to extensive genome-wide changes in DNA methylation. PLoS One. 2013;8(5):e63812.

26. Vogel CFA, Haarmann-Stemmann T. The aryl hydrocarbon receptor repressor-more than a simple feedback inhibitor of AhR signaling: clues for its role in inflammation and cancer. Curr Opin Toxicol. 2017:2:109-19.

27. Buro-Auriemma L, Salit J, Hackett NR, Walters MS, Strulovici-Barel Y, Staudt $M R$, et al. Cigarette smoking induces small airway epithelial epigenetic changes with corresponding modulation of gene expression. Hum Mol Genet. 2013;22(23):4726-38.

28. Squier CA, Kremer MJ. Biology of oral mucosa and esophagus. J Natl Cancer Inst Monogr. 2001;2001(29):7-15.

29. Bergmeier LA. Oral mucosa in health and disease a concise handbook. 2018.

30. Lesch CA, Squier CA, Cruchley A, Williams DM, Speight P. The permeability of human oral mucosa and skin to water. J Dent Res. 1989:68(9):1345-9.

31. Winning TA, Townsend GC. Oral mucosal embryology and histology. Clin Dermatol. 2000;18(5):499-511.

32. Gao X, Jia M, Zhang Y, Breitling LP, Brenner H. DNA methylation changes of whole blood cells in response to active smoking exposure in adults: a systematic review of DNA methylation studies. Clin Epigenetics. 2015;7:113.

33. Sutter TR, Tang YM, Hayes CL, Wo YY, Jabs EW, Li X, et al. Complete cDNA sequence of a human dioxin-inducible mRNA identifies a new gene subfamily of cytochrome P450 that maps to chromosome 2. J Biol Chem. 1994;269(18):13092-9.

34. Hopper JL, Seeman E. The bone density of female twins discordant for tobacco use. N Engl J Med. 1994;330(6):387-92.

35. Law MR, Hackshaw AK. A meta-analysis of cigarette smoking, bone minera density and risk of hip fracture: recognition of a major effect. BMJ. 1997; 315(7112):841-6.

36. Szulc P, Garnero P, Claustrat B, Marchand F, Duboeuf F, Delmas PD. Increased bone resorption in moderate smokers with low body weight: the Minos study. J Clin Endocrinol Metab. 2002;87(2):666-74.

37. Iqbal J, Sun L, Cao J, Yuen T, Lu P, Bab I, et al. Smoke carcinogens cause bone loss through the aryl hydrocarbon receptor and induction of Cyp1 enzymes. Proc Natl Acad Sci U S A. 2013;110(27):11115-20.

38. Yu J, Liu Y, Gong Z, Zhang S, Guo C, Li X, et al. Overexpression long noncoding RNA LINC00673 is associated with poor prognosis and promotes invasion and metastasis in tongue squamous cell carcinoma. Oncotarget. 2017:8(10):16621-32.

39. Spivack SD, Hurteau GJ, Jain R, Kumar SV, Aldous KM, Gierthy JF, et al. Geneenvironment interaction signatures by quantitative mRNA profiling in exfoliated buccal mucosal cells. Cancer Res. 2004;64(18):6805-13.

40. Spira A, Beane J, Shah V, Liu G, Schembri F, Yang X, et al. Effects of cigarette smoke on the human airway epithelial cell transcriptome. Proc Natl Acad Sci U S A. 2004;101(27):10143-8.

41. Cornelis MC, Monda KL, Yu K, Paynter N, Azzato EM, Bennett SN, et al, Genome-wide meta-analysis identifies regions on 7p21 (AHR) and 15q24 (CYP1A2) as determinants of habitual caffeine consumption. PLoS Genet. 2011;7(4):e1002033.

42. Pradhan S, Nagashri MN, Gopinath KS, Kumar A. Expression profiling of CYP1B1 in oral squamous cell carcinoma: counterintuitive downregulation in tumors. PLoS One. 2011;6(11):e27914.

43. Murray GI, Taylor MC, McFadyen MC, McKay JA, Greenlee WF, Burke MD, et al. Tumor-specific expression of cytochrome P450 CYP1B1. Cancer Res. 1997; 57(14):3026-31.

44. Chang I, Mitsui Y, Kim SK, Sun JS, Jeon HS, Kang JY, et al. Cytochrome P450 1B1 inhibition suppresses tumorigenicity of prostate cancer via caspase-1 activation. Oncotarget. 2017:8(24):39087-100.

45. Brand RW, Isselhard DE. Anatomy of orofacial structures : a comprehensive approach. Eight edition. ed. St. Louis: Elsevier; 2019.

46. Aryee MJ, Jaffe AE, Corrada-Bravo H, Ladd-Acosta C, Feinberg AP, Hansen $K D$, et al. Minfi: a flexible and comprehensive Bioconductor package for the analysis of Infinium DNA methylation microarrays. Bioinformatics. 2014; 30(10):1363-9.

47. Smyth GK, Michaud J, Scott HS. Use of within-array replicate spots for assessing differential expression in microarray experiments. Bioinformatics. 2005;21(9):2067-75.
48. Pidsley R, Zotenko E, Peters TJ, Lawrence MG, Risbridger GP, Molloy P, et al. Critical evaluation of the Illumina MethylationEPIC BeadChip microarray for whole-genome DNA methylation profiling. Genome Biol. 2016;17(1):208.

49. McCartney DL, Walker RM, Morris SW, McIntosh AM, Porteous DJ, Evans KL. Identification of polymorphic and off-target probe binding sites on the Illumina Infinium MethylationEPIC BeadChip. Genom Data. 2016;9:22-4.

50. Du P, Zhang X, Huang CC, Jafari N, Kibbe WA, Hou L, et al. Comparison of Beta-value and $\mathrm{M}$-value methods for quantifying methylation levels by microarray analysis. BMC Bioinformatics. 2010;11:587.

51. Fortin JP, Labbe A, Lemire M, Zanke BW, Hudson TJ, Fertig EJ, et al. Functional normalization of 450k methylation array data improves replication in large cancer studies. Genome Biol. 2014;15(12):503.

52. Manimaran S, Selby HM, Okrah K, Ruberman C, Leek JT, Quackenbush J, et al. BatchQC: interactive software for evaluating sample and batch effects in genomic data. Bioinformatics. 2016;32(24):3836-8.

53. Benjamini $Y$, Hochberg Y. Controlling the false discovery rate - a practical and powerful approach to multiple testing. J R Stat Soc Ser B Methodol. 1995;57(1):289-300.

54. Zheng SC, Webster AP, Dong D, Feber A, Graham DG, Sullivan R, et al. A novel cell-type deconvolution algorithm reveals substantial contamination by immune cells in saliva, buccal and cervix. Epigenomics. 2018;10(7):925-40.

55. Patro R, Duggal G, Love MI, Irizarry RA, Kingsford C. Salmon provides fast and bias-aware quantification of transcript expression. Nat Methods. 2017; 14(4):417-9.

56. Soneson C, Love Ml, Robinson MD. Differential analyses for RNA-seq transcript-level estimates improve gene-level inferences. F1000Res. 2015; 4:1521.

57. Love Ml, Huber W, Anders S. Moderated estimation of fold change and dispersion for RNA-seq data with DESeq2. Genome Biol. 2014;15(12):550

58. Liberzon A, Birger C, Thorvaldsdottir H, Ghandi M, Mesirov JP, Tamayo P. The molecular signatures database (MSigDB) hallmark gene set collection. Cell Syst. 2015;1(6):417-25.

59. Subramanian A, Tamayo P, Mootha VK, Mukherjee S, Ebert BL, Gillette MA, et al. Gene set enrichment analysis: a knowledge-based approach for interpreting genome-wide expression profiles. Proc Natl Acad Sci U S A. 2005:102(43):15545-50.

60. Luo W, Friedman MS, Shedden K, Hankenson KD, Woolf PJ. GAGE: generally applicable gene set enrichment for pathway analysis. BMC Bioinformatics. 2009;10:161.

61. Gellner CA, Reynaga DD, Leslie FM. Cigarette smoke extract: a preclinical model of tobacco dependence. Curr Protoc Neurosci. 2016;77:9 54 1-9 10.

62. Cancer Genome Atlas Research N, Weinstein JN, Collisson EA, Mills GB, Shaw $\mathrm{KR}$, Ozenberger BA, et al. The cancer genome Atlas pan-cancer analysis project. Nat Genet. 2013;45(10):1113-20

63. Leek JT, Johnson WE, Parker HS, Jaffe AE, Storey JD. The sva package for removing batch effects and other unwanted variation in high-throughput experiments. Bioinformatics. 2012:28(6):882-3.

\section{Ready to submit your research? Choose BMC and benefit from}

- fast, convenient online submission

- thorough peer review by experienced researchers in your field

- rapid publication on acceptance

- support for research data, including large and complex data types

- gold Open Access which fosters wider collaboration and increased citations

- maximum visibility for your research: over $100 \mathrm{M}$ website views per year

At BMC, research is always in progress.

Learn more biomedcentral.com/submissions 